TRANSACTIONS OF THE

AMERICAN MATHEMATICAL SOCIETY

Volume 356, Number 5, Pages 1971-1999

S 0002-9947(03)03364-6

Article electronically published on October 28, 2003

\title{
ON RESTRICTIONS OF MODULAR SPIN REPRESENTATIONS OF SYMMETRIC AND ALTERNATING GROUPS
}

\author{
ALEXANDER S. KLESHCHEV AND PHAM HUU TIEP
}

\begin{abstract}
Let $\mathbb{F}$ be an algebraically closed field of characteristic $p$ and $H$ be an almost simple group or a central extension of an almost simple group. An important problem in representation theory is to classify the subgroups $G$ of $H$ and $\mathbb{F} H$-modules $V$ such that the restriction $V \downarrow_{G}$ is irreducible. For example, this problem is a natural part of the program of describing maximal subgroups in finite classical groups. In this paper we investigate the case of the problem where $H$ is the Schur's double cover $\hat{A}_{n}$ or $\hat{S}_{n}$.
\end{abstract}

\section{INTRODUCTION}

Let $\mathbb{F}$ be an algebraically closed field of characteristic $p$ and $H$ be an almost simple group or a central extension of an almost simple group. An important problem in representation theory is to classify the subgroups $G$ of $H$ and $\mathbb{F} H$ modules $V$ such that the restriction $V \downarrow_{G}$ is irreducible. For example, this problem is a natural part of the program of describing maximal subgroups in finite classical groups; see [1, 23, 27.

In this paper we investigate the case of the problem where $\operatorname{soc}(H / Z(H))$ is the alternating group $A_{n}$. Assume first that the center $Z(H)$ is trivial, i.e. $H=A_{n}$ or $S_{n}$. In this case Saxl [30] has classified all pairs $(G, V)$ as above, provided the ground field $\mathbb{F}$ has characteristic 0 . In positive characteristic the same has been achieved in [25, 7, 26], at least if $p \neq 2,3$ (and we have obtained a lot of partial information even in the exceptional cases $p=2,3)$.

Assume from now on that $Z(H)$ is non-trivial. If $n \neq 6,7$, then the only nontrivial central extensions are the Schur's double covers $\hat{A}_{n}, \hat{S}_{n}$ and $\tilde{S}_{n}$. So we may and will assume from now on that $p \neq 2$, as otherwise the center acts trivially and so the problem reduces to the case $Z(H)=1$. Moreover, the group algebras $\mathbb{F} \hat{S}_{n}$ and $\mathbb{F} \tilde{S}_{n}$ are canonically isomorphic, so we only have to deal with $\hat{A}_{n}$ and $\hat{S}_{n}$.

To be more precise, $\hat{S}_{n}$ is the double cover of the symmetric group $S_{n}$, in which transpositions lift to involutions. It can be described as the group generated by

Received by the editors October 30, 2002 and, in revised form, April 4, 2003.

2000 Mathematics Subject Classification. Primary 20C20, 20C30, 20C25; Secondary 20B35, 20B20.

Key words and phrases. Representation theory, finite groups.

The authors gratefully acknowledge the support of the NSF (grants DMS-0139019 and DMS0070647). 
$t_{1}, t_{2}, \ldots, t_{n-1}, z$ subject to the following relations:

$$
\begin{aligned}
& z^{2}=1=t_{i}^{2} \quad(1 \leq i \leq n-1), \\
& t_{i} t_{i+1} t_{i}=t_{i+1} t_{i} t_{i+1} \quad(1 \leq i \leq n-2), \\
& t_{i} t_{j}=z t_{j} t_{i} \quad(1 \leq i, j \leq n-1 \text { and }|i-j|>1) .
\end{aligned}
$$

Then $z \in \hat{S}_{n}$ is a central element of order 2 , and we have the following exact sequence:

$$
1 \longrightarrow\langle z\rangle \longrightarrow \hat{S}_{n} \stackrel{\pi}{\longrightarrow} S_{n} \longrightarrow 1 .
$$

For any subgroup $G<S_{n}$ we denote $\hat{G}:=\pi^{-1}(G)$, where $\pi: \hat{S}_{n} \rightarrow S_{n}$ is the natural projection. For example, we have $\hat{A}_{n}$ for the alternating group $A_{n}<S_{n}$.

The results of Kleidman and Wales 22 deal with the problem of irreducible restrictions from $\hat{S}_{n}$ and $\hat{A}_{n}$ to subgroups under the assumption that the ground field has characteristic 0 . In this paper we study the situation in characteristic $p$.

In $\S 3$ we get our first main result: a lower boundary for dimensions of faithful $\mathbb{F} \hat{S}_{n}$ and $\mathbb{F} \hat{A}_{n}$-modules. This turns out to be an effective tool for studying irreducible restrictions. In positive characteristic our result refines the Wagner's lower bound 31. Set

$$
\begin{aligned}
& \delta\left(\hat{S}_{n}\right)= \begin{cases}2^{k-1} & \text { if } n=2 k, \\
2^{k-1} & \text { if } n=2 k+1 \text { and } p \mid n, \\
2^{k} & \text { if } n=2 k+1 \text { and } p \not n ;\end{cases} \\
& \delta\left(\hat{A}_{n}\right)= \begin{cases}2^{k-2} & \text { if } n=2 k \text { and } p \mid n, \\
2^{k-1} & \text { if } n=2 k \text { and } p \not n, \\
2^{k-1} & \text { if } n=2 k+1 .\end{cases}
\end{aligned}
$$

Setting $\kappa_{n}=1$ if $p \mid n$ and 0 otherwise, we have

$$
\delta\left(\hat{S}_{n}\right)=2^{\left\lfloor\left(n-1-\kappa_{n}\right) / 2\right\rfloor} \quad \text { and } \delta\left(\hat{A}_{n}\right)=2^{\left\lfloor\left(n-2-\kappa_{n}\right) / 2\right\rfloor} .
$$

Let $H=\hat{S}_{n}$ or $\hat{A}_{n}$. It is known (see Lemma 2.1 below) that $\delta(H)$ is the dimension of a basic spin $\mathbb{F} H$-module. The following theorem shows that $\delta(H)$ is actually the minimal possible dimension of a faithful $\mathbb{F} H$-module, and there are no other faithful modules with dimensions in the interval $[\delta(H), 2 \delta(H))$.

Theorem A. Let $n \geq 8, H=\hat{S}_{n}$ or $\hat{A}_{n}$, and $V$ be an irreducible faithful $\mathbb{F} H$ module of dimension less than $2 \delta(H)$. Then $V$ is a basic spin module and $\operatorname{dim} V=$ $\delta(H)$.

Our next main theorem classifies the irreducible restrictions from $\hat{S}_{n}$ and $\hat{A}_{n}$ to subgroups $G$ such that $\pi(G)$ is a primitive subgroup of $S_{n}$. We refer the reader to \$2 for the definition of the second basic module.

Theorem B. Let $H=\hat{S}_{n}$ or $\hat{A}_{n}$ with $n \geq 5$, let $D$ be a faithful irreducible $\mathbb{F} H$ module, and let $G$ be a subgroup of $H$ such that $\pi(G)<S_{n}$ is a primitive subgroup which does not contain $A_{n}$. Then $D \downarrow_{G}$ is irreducible if and only if one of the following holds:

(i) $H=\hat{S}_{n}, D$ is a basic spin representation, and one of the following holds:

(a) $n=5, p \neq 5$, and $\pi(G)=\mathbb{Z}_{5}: \mathbb{Z}_{4}$;

(b) $n=6$, and $\pi(G)=S_{5}$;

(c) $n=6, p \neq 3$, and $\pi(G)=A_{5}$;

(d) $n=8$, and $\pi(G)=A G L_{3}(2)$; 
(e) $n=10, p \neq 3,5$, and $\pi(G)=S_{6}, M_{10}$ or $\operatorname{Aut}\left(A_{6}\right)$;

(f) $n=11, p=11$, and $\pi(G)=M_{11}$ (two classes);

(g) $n=12, p \neq 3$, and $\pi(G)=M_{12}$.

(ii) $H=\hat{A}_{n}, D$ is a basic spin representation, and one of the following holds:

(a) $n=5, p \neq 5$, and $\pi(G)=\mathbb{Z}_{5}: \mathbb{Z}_{2}$;

(b) $n=6$, and $\pi(G)=A_{5}$;

(c) $n=7$, and $\pi(G)=L_{2}(7)$ (two classes);

(d) $n=8$, and $\pi(G)=A G L_{3}(2)$ (two classes);

(e) $n=9, p \neq 3$, and $3^{2}: Q_{8} \leq \pi(G) \leq 3^{2}: S L_{2}(3)$ or $L_{2}(8) \leq \pi(G) \leq$ $\operatorname{Aut}\left(L_{2}(8)\right)$

(f) $n=10, p \neq 3$, and $\pi(G)=M_{10}$;

(g) $n=10, p=5$, and $\pi(G)=A_{6}$;

(h) $n=11, p \neq 3$, and $\pi(G)=M_{11}$ (two classes);

(i) $n=12, p \neq 3$, and $\pi(G)=M_{12}$ (two classes).

(iii) $H=\hat{A}_{n}, D$ is a second basic spin representation, and one of the following holds:

(a) $n=6, p=3$, and $\pi(G)=A_{5}$;

(b) $n=8, p \neq 7$, and $\pi(G)=A G L_{3}(2)$ (two classes);

(c) $n=12, p \neq 3,11$, and $\pi(G)=M_{12}$ (two classes).

(iv) $D$ is neither a basic nor a second basic spin representation, and one of the following holds:

(a) $n=5, p \neq 3,5, H=\hat{S}_{5}, \pi(G)=\mathbb{Z}_{5}: \mathbb{Z}_{4}$, and $\operatorname{dim}(D)=4$;

(b) $n=6, p \neq 3,5, H=\hat{S}_{6}, \pi(G)=S_{5}$, and $\operatorname{dim}(D)=4$;

(c) $n=7, p=3, H=\hat{A}_{7}, \pi(G)=L_{2}(7)$ (two classes), and $\operatorname{dim}(D)=6$.

Theorem B is proved in $\$ 5$ In 86 we treat the nearly simple case; that is, we classify the triples $(G, H, D)$ such that $H=\hat{S}_{n}$ or $\hat{A}_{n}, G<H$ with $\operatorname{soc}(G / Z(G))$ simple, $D$ is a faithful $\mathbb{F} H$-module, and $D \downarrow_{G}$ is irreducible. This is the most interesting case for applications to maximal subgroups in finite classical groups. We note that the case $p=0$ has not been treated completely in [22]: Theorem 1.3 of [22] assumes that $G / Z(G)$ is simple. The missing information can be recovered from Theorem $\mathrm{C}$ below by looking at the cases where $p>n$.

To state Theorem $\mathrm{C}$, we need to introduce a delicate combinatorics and recall some results from 9]. For any $n \geq 0$, let $\lambda=\left(\lambda_{1}, \lambda_{2}, \ldots\right)$ be a partition of $n$. We call $\lambda$ a $p$-strict partition if $p$ divides $\lambda_{r}$ whenever $\lambda_{r}=\lambda_{r+1}$. Let $\mathcal{P}_{p}(n)$ denote the set of all $p$-strict partitions of $n$. We say that $\lambda \in \mathcal{P}_{p}(n)$ is restricted if

$$
\begin{cases}\lambda_{r}-\lambda_{r+1}<p & \text { if } p \mid \lambda_{r} \\ \lambda_{r}-\lambda_{r+1} \leq p & \text { if } p \nmid \lambda_{r}\end{cases}
$$

for each $r \geq 1$. Let $\mathcal{R} \mathcal{P}_{p}(n)$ denote the set of all restricted $p$-strict partitions of $n$.

Let $\lambda \in \mathcal{P}_{p}(n)$. We identify $\lambda$ with its Young diagram

$$
\lambda=\left\{(r, s) \in \mathbb{Z}_{>0} \times \mathbb{Z}_{>0} \mid s \leq \lambda_{r}\right\} .
$$

Elements $(r, s) \in \mathbb{Z}_{>0} \times \mathbb{Z}_{>0}$ are called nodes. Define $\ell:=(p-1) / 2$, and label the nodes of $\lambda$ with residues, which are the elements of the set $I=\{0,1, \ldots, \ell\}$. The labelling depends only on the column and follows the repeating pattern $0,1, \ldots$, $\ell-1, \ell, \ell-1, \ldots, 1,0$, starting from the first column and going to the right; see the example below. The residue of the node $A$ is denoted res $A$. Denote by $a(\lambda)$ the number of nodes in the Young diagram $\lambda$ of residue different from 0 . 
Let $i \in I$. A node $A=(r, s) \in \lambda$ is called $i$-removable (for $\lambda$ ) if one of the following holds:

(R1) res $A=i$ and $\lambda-\{A\}$ is again a $p$-strict partition;

(R2) the node $B=(r, s+1)$ immediately to the right of $A$ belongs to $\lambda$, res $A=$ res $B=i$, and $\lambda-\{A, B\}$ is a $p$-strict partition.

Similarly, a node $B=(r, s) \notin \lambda$ is called $i$-addable (for $\lambda$ ) if one of the following holds:

(A1) res $B=i$ and $\lambda \cup\{B\}$ is again a $p$-strict partition;

(A2) the node $A=(r, s-1)$ immediately to the left of $B$ does not belong to $\lambda$, res $A=\operatorname{res} B=i$, and $\lambda \cup\{A, B\}$ is a $p$-strict partition.

We note that (R2) and (A2) above are only possible in case $i=0$.

Now label all $i$-addable nodes of the diagram $\lambda$ by + and all $i$-removable nodes by -. Then, the $i$-signature of $\lambda$ is the sequence of pluses and minuses obtained by going along the rim of the Young diagram from bottom left to top right and reading off all the signs. The reduced $i$-signature of $\lambda$ is obtained from the $i$-signature by successively erasing all neighboring pairs of the form +- . Note the reduced $i$-signature always looks like a sequence of -'s followed by +'s. Nodes corresponding to a - in the reduced $i$-signature are called $i$-normal. The rightmost $i$-normal node (corresponding to the rightmost - in the reduced $i$-signature) is called $i$-good. If $A$ is the $i$-good node, we write $\lambda_{[i]}$ for $\lambda-\{A\}$. A node is called normal if it is $i$-normal for some $i$.

Example. Let $p=5$, so $\ell=2$. The partition $\lambda=(16,11,10,10,9,5,1)$ is restricted 5 -strict, and its residues are as follows:

\begin{tabular}{|c|c|c|c|c|c|c|c|c|c|c|c|c|}
\hline 0 & 1 & 2 & 1 & 0 & 0 & 1 & 2 & 1 & 0 & 0 & \begin{tabular}{l|l|}
1 & 2 \\
\end{tabular} & \begin{tabular}{l|l|l|}
1 & 0 & 0 \\
\end{tabular} \\
\hline 0 & 1 & 2 & 1 & 0 & 0 & 1 & 2 & 1 & 0 & 0 & & \\
\hline 0 & 1 & 2 & 1 & 0 & 0 & 1 & 2 & 1 & 0 & & & \\
\hline 0 & 1 & 2 & 1 & 0 & 0 & 1 & 2 & 1 & 0 & & & \\
\hline 0 & 1 & 2 & 1 & 0 & 0 & 1 & 2 & 1 & & & & \\
\hline 0 & 1 & 2 & 1 & 0 & & & & & & & & \\
\hline 0 & & & & & & & & & & & & \\
\hline
\end{tabular}

The 0-addable and 0-removable nodes are as labelled in the diagram:

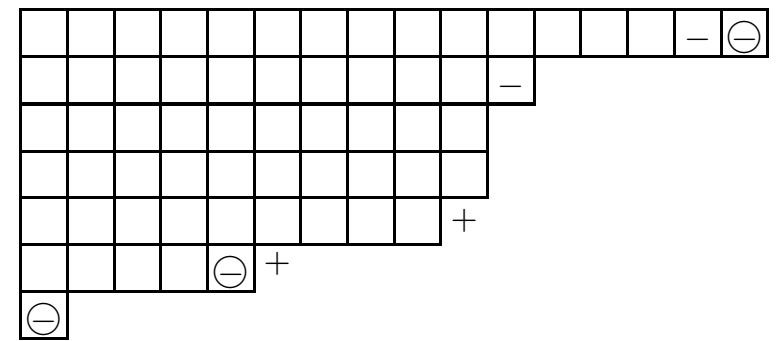

Hence, the 0 -signature of $\lambda$ is,,,,,,--++--- and the reduced 0 -signature is ,,--- . The 0 -normal nodes are circled in the diagram above.

It is proved in [9] that the irreducible $\mathbb{F} \hat{S}_{n}$-modules are labelled by the symbols $(\lambda, \sigma)$ where $\lambda$ runs over all restricted $p$-strict partitions of $n, \sigma$ is 0 if $a(\lambda)$ is even and $\sigma$ runs over $\{+,-\}$ if $a(\lambda)$ is odd. The irreducible module corresponding to a 
pair $(\lambda, \sigma)$ is denoted by $D(\lambda, \sigma)$. Moreover, we have $D(\lambda, 0) \otimes \operatorname{sgn} \cong D(\lambda, 0)$ and $D(\lambda, \pm) \otimes \mathbf{s g n} \cong D(\lambda, \mp)$, so Clifford theory yields the following. If $\lambda \in \mathcal{R P}_{p}(n)$ has $a(\lambda)$ even, then $D(\lambda, 0) \downarrow_{\hat{A}_{n}}$ decomposes as a direct sum $E(\lambda,+) \oplus E(\lambda,-)$ of two non-isomorphic irreducible $\mathbb{F} \hat{A}_{n}$-modules. If $a(\lambda)$ is odd, then $D(\lambda,+) \downarrow_{\hat{A}_{n}} \cong$ $D(\lambda,-) \downarrow_{\hat{A}_{n}}$ is irreducible, denoted $E(\lambda, 0)$. Now,

$$
\left\{E(\lambda, 0) \mid \lambda \in \mathcal{R} \mathcal{P}_{p}(n), a(\lambda) \text { odd }\right\} \sqcup\left\{E(\lambda,+), E(\lambda,-) \mid \lambda \in \mathcal{R} \mathcal{P}_{p}(n), a(\lambda) \text { even }\right\}
$$

gives a complete set of pairwise non-isomorphic irreducible $\mathbb{F} \hat{A}_{n}$-modules.

The partition $\omega_{n}$, for which $D\left(\omega_{n}, \sigma\right)$ and $E\left(\omega_{n}, \sigma\right)$ are basic spin modules, can be described as follows; see [9, 9.12]. Write $n=a p+b$ with $0 \leq b<p$. Then

$$
\omega_{n}:= \begin{cases}\left(p^{a}, b\right) & \text { if } b \neq 0 \\ \left(p^{a-1}, p-1,1\right) & \text { if } b=0 .\end{cases}
$$

Let $\lambda \in \mathcal{R P}_{p}(n)$. We write $\lambda \in J S$ if the bottom removable node of $\lambda$ is its only normal node ( $J S$ stands for Jantzen-Seitz; cf. 18, 24). Let $i \in I$. We write $\lambda \in J S(i)$ if $\lambda \in J S$ and the bottom removable node of $\lambda$ has residue $i$.

We set $\Omega:=\{1,2, \ldots, n\}$ and say that a subgroup $X<S_{n}$ is of type

$$
\left(n_{1}, n_{2}, \ldots, n_{t}\right)
$$

if the $X$-orbits on $\Omega$ have lengths $n_{1}, n_{2}, \ldots, n_{t}$.

The following result includes Theorems 9.17, 9.18 from [9] and some results from [29] (all of which are used in the proof).

Theorem C. Let $H=\hat{S}_{n}$ or $\hat{A}_{n}$, D be a faithful irreducible $\mathbb{F} H$-module, and $G$ be a subgroup of $H$ such that $G / Z(G)$ is almost simple and $\pi(G)$ does not contain $A_{n}$. Set $S:=\operatorname{soc}(\pi(G))$. Then $D \downarrow_{G}$ is irreducible if and only if one of the following holds:

(i) $S=A_{m}(m \geq 5), n=m+1$, and one of the following holds (all embeddings being of type $(m, 1))$ :

(a) $H=\hat{S}_{m+1}, G=\hat{S}_{m}$, and either $D=D(\lambda, 0)$ for some $\lambda \in J S(0)$ with $a(\lambda)$ even or $D=D(\lambda, \pm)$ for some $\lambda \in J S$ with $a(\lambda)$ odd.

(b) $H=\hat{S}_{m+1}, G=\hat{A}_{m}$, and $D=D(\lambda, \pm)$ for some $\lambda \in J S(0)$ with $a(\lambda)$ odd.

(c) $H=\hat{A}_{m+1}, G=\hat{A}_{m}$, and either $D=E(\lambda, 0)$ for some $\lambda \in J S(0)$ with $a(\lambda)$ odd or $D=E(\lambda, \pm)$ for some $\lambda \in J S$ with $a(\lambda)$ even.

(ii) $S=A_{m}(m \geq 5), n=m+2$, and one of the following holds:

(a) $H=\hat{S}_{m+2}, G=\hat{S}_{m}$ (embedding of type $(m, 1,1)$ ), and $D=D(\lambda, \pm$ ) for some $\lambda \in J S(0)$ with $a(\lambda)$ odd, such that $\lambda_{[0]} \in J S(1)$.

(b) $H=\hat{A}_{m+2}, \pi(G)=\left(S_{m} \times S_{2}\right) \cap A_{m+2} \cong S_{m}$ (embedding of type $(m, 2))$, and one of the following holds:

(1) $D=E(\lambda, \sigma)$ for some $\lambda \in J S(0)$ such that $\lambda_{[0]} \in J S(1)$.

(2) $D$ is basic spin, $m$ is odd, and $m \not \equiv-2,0(\bmod p)$.

(c) $H=\hat{S}_{m+2}, \pi(G)=\left(S_{m} \times S_{2}\right) \cap A_{m+2} \cong S_{m}$ (embedding of type $(m, 2))$, and $D=D(\lambda, \pm)$ for some $\lambda \in J S(0)$ with $a(\lambda)$ odd such that $\lambda_{[0]} \in J S(1)$.

(d) $H=\hat{A}_{m+2}, G=\hat{A}_{m}$ (embedding of type $(m, 1,1)$ ), and $D=E(\lambda, \pm$ ) for some $\lambda \in J S(0)$ with $a(\lambda)$ even such that $\lambda_{[0]} \in J S(1)$. 
(iii) $S=A_{m}(m \geq 5), n=m+3,3 \neq p \mid n, m$ is odd, $H=\hat{A}_{m+3}, \pi(G)=$ $\left(S_{m} \times S_{2} \times S_{1}\right) \cap A_{m+3} \cong S_{m}$ of type $(m, 2,1)$, and $D$ is basic spin.

(iv) $\pi(G)$ is primitive on $\Omega, S \in\left\{A_{5}, A_{6}, L_{2}(7), L_{2}(8), M_{11}, M_{12}\right\}$, and the triple $(G, H, D)$ is as listed in Theorem $B$.

(v) $S=A_{5}, n=7, \pi(G)$ is of type $(6,1)$, and one of the following holds:

(a) $H=\hat{A}_{7}, \pi(G)=A_{5}$, and either $p=3$ and $D$ is second basic (of dimension 6$)$, or $p \neq 3$ and $D$ is basic spin.

(b) $H=\hat{S}_{7}, p=7, \pi(G)=A_{5}$ or $S_{5}$, and $D$ is basic spin.

(vi) $S=A_{6}, n=11, D$ is basic spin, and $\pi(G)$ is of type $(10,1)$. If $H=\hat{A}_{11}$, then $p \neq 3,5$ and $\pi(G)=M_{10}$. If $H=\hat{S}_{11}$, then $p=11$ and $\pi(G)=M_{10}$, $S_{6}$, or $\operatorname{Aut}\left(A_{6}\right)$.

(vii) $S=L_{2}(8), H=\hat{A}_{10}, p=5, \pi(G)=S$ or $\operatorname{Aut}(S), \pi(G)$ is of type $(9,1)$, and $D$ is basic spin.

(viii) $S=M_{12}, D$ is basic spin, and one of the following holds:

(a) $n=13, \pi(G)=S$ is of type $(12,1)$, and $(H, p)=\left(\hat{S}_{13}, 13\right)$ or $\left(\hat{A}_{13}, \neq\right.$ $3)$;

(b) $n=14, \pi(G)=S$ is of type $(12,1,1)$, and $(H, p)=\left(\hat{A}_{14}, 7\right)$.

Finally, we consider subgroups $G$ of $\hat{S}_{n}$ and $\hat{A}_{n}$ with $\pi(G)$ imprimitive and not almost simple. Here we have the following results.

Theorem D. Let $p>3, n \geq 7$ and $H=\hat{S}_{n}$ or $\hat{A}_{n}$. Suppose that $G<H$ is a subgroup with $\pi(G)$ imprimitive on $\Omega$, and such that $G / Z(G)$ is not almost simple. Assume further that $D$ is a faithful non-basic $\mathbb{F} H$-module such that $D \downarrow_{G}$ is irreducible. Then $\pi(G)$ is transitive, $n$ is even, and one of the following happens:

(i) $\pi(G)$ has 2 blocks of imprimitivity of size $n / 2$.

(ii) $\pi(G)$ has $n / 2$ blocks of imprimitivity of size 2 .

We believe that even in the exceptional cases of Theorem $\mathrm{D}$ the restriction is usually reducible (with very few exceptions, like the one in [22, Theorem 1.1(6)]), and that the restriction $p>3$ is unnecessary, but we cannot prove this at the moment. Note also that we have excluded basic modules in Theorem D. The following result describes the irreducible restrictions of basic modules to maximal imprimitive subgroups. We refer the reader to $\$$ f for more results on basic modules.

Theorem E. Let $H=\hat{S}_{n}$ or $\hat{A}_{n}$, let $D$ be a basic spin $\mathbb{F} H$-module, and let $G<H$ be a subgroup with $\pi(G)$ maximal imprimitive. Then $D \downarrow_{G}$ is irreducible if and only if one of the following holds:

(i) $H=\hat{S}_{n}$ and one of the following holds:

(a) $\pi(G)=S_{n-a} \times S_{a}, a<n / 2, p \nmid a, p \nmid(n-a)$, and either $n$ is even, or $n$ is odd and $p \mid n$.

(b) $\pi(G)=S_{a} \prec S_{b}$ for some $a, b \geq 2$ with $n=a b$ and $p \nmid a$.

(ii) $H=\hat{A}_{n}$ and one of the following holds:

(a) $\pi(G)=A_{n} \cap\left(S_{n-a} \times S_{a}\right), a<n / 2, p \nmid a, p \nmid(n-a)$, and either $n$ is odd, or $n$ is even and $p \mid n$.

(b) $\pi(G)=A_{n} \cap\left(S_{a} \curlywedge S_{b}\right)$ for some $a, b \geq 2$ with $n=a b$ and $p \nmid a$.

Finally we mention a result from 98 on irreducible tensor products, which is also relevant to the problem on maximal subgroups and follows from the results of $\$[7$ Similar results for symmetric and alternating groups in arbitrary characteristic or 
Schur's double covers in characteristic 0 were obtained in [4, 5, 6] 3, 13. In the next theorem $h(\lambda)$ denotes the number of non-zero parts of a partition $\lambda$ and $\lambda^{\mathbf{M}}$ is the Mullineux image of $\lambda$.

Theorem F. Let $p>3$ and $n \geq 7$.

(i) Let $H=\hat{S}_{n}$ or $\hat{A}_{n}$, and $V, W$ be non-basic faithful $\mathbb{F} H$-modules. Then $V \otimes W$ is reducible.

(ii) Let $V$ be a non-basic faithful $\mathbb{F} \hat{S}_{n}$-module, and $W=D^{\lambda}$ be an irreducible $\mathbb{F} S_{n}$-module with $h(\lambda)>2$ and $h\left(\lambda^{\mathbf{M}}\right)>2$. Then $V \otimes W$ is reducible.

\section{Preliminaries}

Recall that $\mathbb{F}$ is an algebraically closed field of characteristic $p \neq 2$. For any group $G$ we denote by $\mathbf{1}_{G}$ or just $\mathbf{1}$ the trivial $\mathbb{F} G$-module. Also denote by sgn the 1-dimensional sign representation of the symmetric group $S_{n}$.

If $g \in S_{n}$ is any element, we denote by $\hat{g}$ any element in $\pi^{-1}(g)$. Thus, $\pi^{-1}(g)=$ $\{\hat{g}, z \hat{g}\}$. Also, if $g \in S_{n}$ is an element of odd order, then we can write $\pi^{-1}(g)=$ $\left\{g^{+}, g^{-}\right\}$, where $g^{+}$has the same order as $g$ and $g^{-}=z g^{+}$.

For any composition $\lambda$ of $n$ we denote the corresponding Young subgroup of $S_{n}$ by $S_{\lambda}$ and $A_{\lambda}:=S_{\lambda} \cap A_{n}$. Set

$$
M^{\lambda}=\left(\mathbf{1}_{\hat{S}_{\lambda}}\right) \uparrow^{\hat{S}_{n}} .
$$

Note that $z$ acts trivially on $M^{\lambda}$. On restriction, $M^{\lambda}$ is also an $\mathbb{F} \hat{A}_{n}$-module, isomorphic to $\left(\mathbf{1}_{\hat{A}_{\lambda}}\right) \uparrow^{\hat{A}_{n}}$.

If $G$ is a finite group and $V$ is a finite dimensional $\mathbb{C} G$-module, we denote by $\bar{V}$ its reduction modulo $p$, considered as an element of the Grothendieck group of $\mathbb{F} G$-modules. If $L_{1}, \ldots, L_{k}$ are irreducible $\mathbb{F} G$-modules, the notation

$$
\bar{V}=a_{1} L_{1}+\cdots+a_{k} L_{k}
$$

means that $\bar{V}=a_{1}\left[L_{1}\right]+\cdots+a_{k}\left[L_{k}\right]$ in the Grothendieck group. We will often abuse the notion and speak of $\bar{V}$ as a module - for example if $\bar{V}$ is irreducible, then we will speak of the irreducible module $\bar{V}$, which is defined up to isomorphism.

Let $G=\hat{S}_{n}$ or $\hat{A}_{n}$. Then $G$ possesses (one or two) basic (spin) modules over $\mathbb{C}$ these are the modules whose characters correspond to the partition $(n)$ in Schur's classification. Composition factors of their reductions modulo $p$ will be called basic (spin) modules over $\mathbb{F} G$. Moreover, the irreducible $\mathbb{C} G$-modules with characters corresponding to the partition $(n-1,1)$ will be referred to as second basic (spin) modules. Composition factors of their reductions modulo $p$, different from basic spin modules, will be referred to as second basic (spin) modules over $\mathbb{F} G$. Basic and second basic modules over $\mathbb{C} G$ and $\mathbb{F} G$ were studied in detail by Wales; see 32. Tables III, IV]. We reproduce this information for basic modules:

Lemma 2.1 ([32, Table III]). Suppose $n \geq 4$.

(i) Let $n$ be even. There are two basic spin modules $Y_{n, \mathbb{C}}^{+}$and $Y_{n, \mathbb{C}}^{-}$over $\mathbb{C} \hat{S}_{n}$ and one basic spin module $U_{n, \mathbb{C}}$ over $\mathbb{C} \hat{A}_{n}$, all of dimension $2^{(n-2) / 2}$, and $Y_{n, \mathbb{C}}^{+} \downarrow_{\hat{A}_{n}} \cong Y_{n, \mathbb{C}}^{-} \downarrow_{\hat{A}_{n}} \cong U_{n, \mathbb{C}}$. Moreover,

(a) If $p \nmid n$, then $Y_{n}^{ \pm}:=\bar{Y}_{n, \mathbb{C}}^{ \pm}$and $U_{n}:=\bar{U}_{n, \mathbb{C}}$ are irreducible. Moreover, $Y_{n}^{+} ¥ Y_{n}^{-}$, and $Y_{n}^{+} \downarrow_{\hat{A}_{n}} \cong Y_{n}^{-} \downarrow_{\hat{A}_{n}} \cong U_{n}$. 
(b) If $p \mid n$, then $Y_{n}:=\bar{Y}_{n, \mathbb{C}}^{+} \cong \bar{Y}_{n, \mathbb{C}}^{-}$is irreducible. Moreover, $Y_{n} \downarrow_{\hat{A}_{n}} \cong$ $U_{n}^{+} \oplus U_{n}^{-}$for irreducible $\hat{A}_{n}$-modules $U_{n}^{+} ¥ U_{n}^{-}$, and $\bar{U}_{n, \mathbb{C}}=U_{n}^{+}+U_{n}^{-}$.

(ii) Let $n$ be odd. There is one basic spin module $Y_{n, \mathbb{C}}$ over $\mathbb{C} \hat{S}_{n}$ and two basic spin modules $U_{n, \mathbb{C}}^{+}$and $U_{n, \mathbb{C}}^{-}$over $\mathbb{C} \hat{A}_{n}$, with $\operatorname{dim} Y_{n, \mathbb{C}}=2 \operatorname{dim} U_{n, \mathbb{C}}^{ \pm}=$ $2^{(n-1) / 2}$, and $Y_{n, \mathbb{C}} \downarrow_{\hat{A}_{n}} \cong U_{n, \mathbb{C}}^{+} \oplus U_{n, \mathbb{C}}^{-}$. Moreover,

(a) If $p \nmid n$, then $Y_{n}:=\bar{Y}_{n, \mathbb{C}}$ and $U_{n}^{ \pm}:=\bar{U}_{n, \mathbb{C}}^{ \pm}$are irreducible. Moreover, $U_{n}^{+} \not U_{n}^{-}$, and $Y_{n} \downarrow_{\hat{A}_{n}} \cong U_{n}^{+} \oplus U_{n}^{-}$.

(b) If $p \mid n$, then $\bar{Y}_{n, \mathbb{C}}$ has two composition factors $Y_{n}^{+} \not Y_{n}^{-}$, and $U_{n}:=$ $\bar{U}_{n, \mathbb{C}}^{-} \cong \bar{U}_{n, \mathbb{C}}^{+}$is irreducible. Moreover, $Y_{n}^{+} \downarrow_{\hat{A}_{n}} \cong Y_{n}^{-} \downarrow_{\hat{A}_{n}} \cong U_{n}$.

Occasionally, while speaking of the basic spin representations $Y_{n, \mathbb{C}}^{( \pm)}, Y_{n}^{( \pm)}$, etc., we will allow for $n=2$ and 3. For such $n$, projective representations of $S_{n}$ and $A_{n}$ are linear, and we use the following interpretations: $Y_{2, \mathbb{C}}^{+}\left(\operatorname{resp} . Y_{2}^{+}\right)$is the trivial $\mathbb{C} S_{3^{-}}$(resp. $\left.\mathbb{F} S_{3^{-}}\right)$module, $Y_{2, \mathbb{C}}^{-}\left(\right.$resp. $\left.Y_{2}^{-}\right)$is the sign $\mathbb{C} S_{3^{-}}$-module, $Y_{3, \mathbb{C}}$ is the natural 2-dimensional $\mathbb{C} S_{3}$-module, $Y_{3}$ is the natural 2-dimensional $\mathbb{F} S_{3}$-module if $p>3$, and $Y_{3}^{+}$and $Y_{3}^{-}$are, respectively, the trivial and the sign modules over $\mathbb{F} S_{3}$ if $p=3$. Observe that under these interpretations the formulas for dimensions in Lemma 2.1 and the facts about reductions modulo $p$ and restrictions to $A_{n}$ still hold.

We recall some other known results. The first one is due to Wagner.

Lemma 2.2 (31]). Let $n \geq 9, s$ be the number of terms in the 2-adic expansion of $n$, and $H=\hat{S}_{n}$ or $\hat{A}_{n}$. Then $2^{\lfloor(n-s-1) / 2\rfloor}$ divides $\operatorname{dim} D$ for any faithful $\mathbb{F} H$-module D.

Lemma 2.2 implies that $\operatorname{dim} D \geq 2^{\lfloor(n-s-1) / 2\rfloor}$ for any faithful $\mathbb{F} H$-module $D$. In $\$ 3$ we prove Theorem A, which improves this lower bound. Next, we cite a result of Wales. If $p \neq 3$, it shows that certain elements of order 3 in $\hat{S}_{n}$ have three different eigenvalues on irreducible modules, except possibly basic spin modules.

Lemma 2.3 ([32, 8.1]). Let $n \geq 5, c=(123)^{+} \in \hat{S}_{n}$, and $V$ be an irreducible $\mathbb{F} \hat{S}_{n}$-module on which $c$ has a quadratic minimal polynomial. Then $V$ is basic spin.

The following 'recognition' result for basic spin representations was also essentially demonstrated by Wales in the proof of [32, 8.1].

Lemma 2.4. Let $n \geq 6$, and let $V$ be an irreducible $\mathbb{F} \hat{S}_{n}$-module (resp. $\mathbb{F} \hat{A}_{n}$ module). Then $V$ is basic spin if and only if all composition factors of the restriction $V \downarrow_{\hat{S}_{n-1}}\left(\right.$ resp. $V \downarrow_{\hat{A}_{n-1}}$ ) are basic spin.

Proof. We prove the result for $\hat{S}_{n}$, the proof for $\hat{A}_{n}$ being similar. By Frobenius reciprocity we may assume that $V$ is a constituent of a reduction modulo $p$ of $\left(Y_{n-1, \mathbb{C}}^{( \pm)}\right) \uparrow \hat{S}_{n}$. By the branching theorem [15, 10.2], this induced module is a sum of basic modules $Y_{n}^{( \pm)}$and second basic modules $Z_{n}^{( \pm)}$. Now if $V$ is not basic, then $V$ has to be a non-basic constituent of reduction $\bar{Z}_{n}^{( \pm)}$. But then it follows from 32 Table IV] that the restriction $V \downarrow_{\hat{S}_{n-1}}$ contains a non-basic constituent, contrary to our assumption. 
Lemma 2.5. Let $n \geq 7, p \neq 2,3$, and $\varphi$ be an irreducible faithful Brauer character of $\hat{S}_{n}$. Define the elements $c, d \in \hat{S}_{n}$ by setting $c:=(123)^{+}$and $d:=((123)(456))^{+}$. Then $\varphi(c)$ and $\varphi(d)$ are rational integers with

(i) $\varphi(1) / 7 \geq \varphi(c) \geq-\varphi(1) / 2$, and $\varphi(c)=-\varphi(1) / 2$ if and only if $\varphi$ is basic spin.

(ii) $\varphi(1) / 4 \geq \varphi(d) \geq-\varphi(1) / 8$, and $\varphi(d)=\varphi(1) / 4$ if and only if $\varphi$ is basic spin. Moreover, if $n \geq 11$, then $\varphi(d) \geq-\varphi(1) / 56$.

Proof. We prove (i), the proof of (ii) being similar. Apply induction on $n$. The statement is true for small $n$ by [19]. For the inductive step, assume (i) holds for $n-1$. Then $\varphi \downarrow_{\hat{S}_{n-1}}=\sum_{i=1}^{t} \varphi_{i}$ for some irreducible faithful Brauer characters $\varphi_{i}$ of $\hat{S}_{n-1}$. By induction hypothesis, $\varphi_{i}(1) / 7 \geq \varphi_{i}(c) \geq-\varphi_{i}(1) / 2$ for each $i$, whence $\varphi(1) / 7 \geq \varphi(c) \geq-\varphi(1) / 2$. Moreover, if $\varphi(c)=-\varphi(1) / 2$, then $\varphi_{i}(c)=-\varphi_{i}(1) / 2$ for each $i$, whence $\varphi_{i}$ is basic by induction hypothesis, and so $\varphi$ itself is basic by Lemma 2.4.

\section{Minimal Dimensions (Theorem A)}

In this section we prove Theorem A. Apply induction on $n$. For small $n$ the statement holds by [19]. Let $n \geq 12$. Assume $V$ is not basic. We have to show that $\operatorname{dim} V \geq 2 \delta(H)$. Let $\varphi$ be the Brauer character of $V$. We will often use the fact that the minimal polynomial of $c$ on $V$ has degree 3; see Lemma 2.3. We consider several cases.

Case S1: $H=\hat{S}_{n}, n=2 k$, and $p \nmid(n-2)$. Note that $C_{H}\left(t_{1}\right) \geq\left\langle t_{1}\right\rangle \times \hat{A}_{n-2}$. Since $t_{1}$ is not central, it has both eigenvalues 1 and -1 on $V$. Let $V_{+}$and $V_{-}$ be the corresponding eigenspaces. We have $t_{3} t_{1} t_{3}=z t_{1}$ and $z=-1$ on $V$, so $t_{3}$ swaps $V_{+}$and $V_{-}$. Observe that $V_{ \pm}$are $\hat{A}_{n-2}$-modules, and $t_{3}$ normalizes $\hat{A}_{n-2}$. In particular, all composition factors of $V_{+}$are basic for $\hat{A}_{n-2}$ if and only if all composition factors of $V_{-}$are basic for $\hat{A}_{n-2}$. But all composition factors of both $V_{+}$and $V_{-}$could not be basic, as then $V$ itself would be basic by Lemma 2.4 Thus both $V_{ \pm}$have non-basic composition factors. By the induction hypothesis, $\operatorname{dim} V_{ \pm} \geq 2 \delta\left(\hat{A}_{n-2}\right)=2^{k-1}$, hence $\operatorname{dim} V \geq 2^{k}=2 \delta\left(\hat{S}_{n}\right)$.

Case S2: $H=\hat{S}_{n}, p \mid n=2 k+1$. Argue as in S1.

Case S3: $H=\hat{S}_{n}, n=2 k, p \mid(n-2)$, and $p \neq 3$. Clearly, $C_{H}(c) \geq\langle c\rangle \times \hat{S}_{n-3}$. By Lemma 2.3 $c$ has all three eigenvalues $\omega^{i}, i=0,1,2$, on $V$, where $\omega \in \mathbb{F}$ is a primitive third root of unity. Let $V_{i}, i=1,2,3$, be the corresponding eigenspaces, considered as modules over $\hat{S}_{n-3}$. Then at least one of them has a non-basic composition factor by Lemma 2.4. Thus

$$
\operatorname{dim} V \geq 4 \delta\left(\hat{S}_{n-3}\right)=4 \cdot 2^{k-2}=2 \delta\left(\hat{S}_{n}\right) .
$$

Case $S_{4}: H=\hat{S}_{n}, n=2 k+1, p \nmid n, p \nmid(n-3)$, and $p \neq 3$. We restrict $V$ to $K:=S_{3} \times \hat{A}_{n-3}$, where $S_{3}$ is generated by $t_{1}$ and $t_{2}$. Since $p \neq 2,3$, there are three irreducible characters of $S_{3}$ : the trivial character $\chi$, the sign character $\psi$, and a character $\alpha$ of degree 2. By Lemma 2.3, $\alpha$ and at least one of $\chi, \psi$ appear in $V \downarrow_{S_{3}}$. Next, $t_{4}$ centralizes $c$ and $t_{4} t_{1} t_{4}=z t_{1}$, hence $t_{4}$ swaps the characters $\chi$ and $\psi$. Thus, all three characters $\chi, \psi$, and $\alpha$ appear in $\varphi \downarrow_{S_{3}}$, and we can write

$$
\varphi \downarrow_{K} \cong \alpha \otimes \beta_{1}+\chi \otimes \beta_{2}+\psi \otimes \beta_{3},
$$


for some Brauer characters $\beta_{1}, \beta_{2}, \beta_{3}$ of $\hat{A}_{n-3}$. Moreover, the characters $\beta_{2}$ and $\beta_{3}$ are $t_{4}$-conjugate. We have $\alpha(c)=-1$ and $\beta_{3}(g)=\beta_{2}\left(t_{4} g t_{4}\right)$ for any $g \in \hat{A}_{n-3}$. In particular, $\beta_{2}(1)=\beta_{3}(1)$. So $\varphi(c)=-\beta_{1}(1)+2 \beta_{2}(1)$. Let $c^{\prime}=(6,7,8)^{+} \in \hat{A}_{n-3}$. As $c^{\prime}$ is conjugate to $c$ and is centralized by $t_{4}$, we get $\varphi(c)=\varphi\left(c^{\prime}\right)=2 \beta_{1}\left(c^{\prime}\right)+2 \beta_{2}\left(c^{\prime}\right)$. Therefore

$$
\beta_{1}(1)+2 \beta_{1}\left(c^{\prime}\right)=2\left(\beta_{2}(1)-\beta_{2}\left(c^{\prime}\right)\right)>0 .
$$

Hence $\beta_{1}$ contains a non-basic irreducible Brauer character by Lemma 2.5. If $\beta_{1}$ is not irreducible, then

$$
\varphi(1) \geq \alpha(1)\left(2 \delta\left(\hat{A}_{n-3}\right)+\delta\left(\hat{A}_{n-3}\right)\right)+2 \delta\left(\hat{A}_{n-3}\right)=2^{k+1}=2 \delta\left(\hat{S}_{n}\right) .
$$

Now, let $\beta_{1}$ be irreducible. If either $\beta_{2}$ is not irreducible or $\beta_{2}$ is not basic, then

$$
\varphi(1) \geq 2 \alpha(1) \delta\left(\hat{A}_{n-3}\right)+4 \delta\left(\hat{A}_{n-3}\right) \geq 2 \delta\left(\hat{S}_{n}\right) .
$$

We are left with the case where all $\beta_{i}$ are irreducible and $\beta_{2}, \beta_{3}$ are basic. Moreover, we may assume that $\beta_{1}(1)<3 \cdot 2^{k-2}$. By Lemma 2.5, the right-hand side of (3.1) equals $3 \cdot 2^{k-2}$, so $\beta_{1}\left(c^{\prime}\right)>0$. As $c c^{\prime}$ is conjugate to the element $d$ from Lemma 2.5. we now have by that lemma:

$$
-\varphi(1) / 8 \leq \varphi(d)=-\beta_{1}\left(c^{\prime}\right)+2 \beta_{2}\left(c^{\prime}\right)<2 \beta_{2}\left(c^{\prime}\right)=-\beta_{2}(1)=-2^{k-2} .
$$

Hence $\varphi(1)>2^{k+1}$.

Case $S 5: H=\hat{S}_{n}, n=2 k+1, p \nmid n, p \mid(n-3)$, and $p \neq 3$. We restrict $V$ to the central product $K:=\hat{A}_{4} * \hat{S}_{n-4}$, where $\hat{S}_{n-4}$ is generated by $t_{j}, 5 \leq j \leq n-1$, and $\hat{A}_{4}$ is generated by $c=t_{1} t_{2}$ and $t_{2} t_{3}$. Observe that $\hat{A}_{4} \cong Q_{8} \cdot\langle c\rangle$ has exactly 3 faithful irreducible characters, say $\alpha_{i}, i=0,1,2$, all of degree 2 , with $\alpha_{i}(c)=-\omega^{i}$ (see also [15, 4.8]). So we can write

$$
\varphi \downarrow_{K}=x \alpha_{1} \otimes \beta_{1}+y \alpha_{2} \otimes \beta_{2}+z \alpha_{3} \otimes \beta_{3}
$$

for some faithful Brauer characters $\beta_{i}$ of $\hat{A}_{n-3}$ and $x, y, z \in\{0,1\}$. Note that $u:=t_{1} t_{5}$ normalizes both $\hat{A}_{4}$ and $\hat{S}_{n-4}$. Also $u c u^{-1}=c^{-1}$, so the characters $\alpha_{2}$ and $\alpha_{3}$ are $u$-conjugate. It follows that the components $y \alpha_{2} \otimes \beta_{2}$ and $z \alpha_{3} \otimes \beta_{3}$ are $u$-conjugate. Now Lemma 2.3 implies that $y=z=1$.

If $\beta_{2}$ is not basic or is not irreducible, then

$$
\operatorname{dim} V \geq 8 \delta\left(\hat{S}_{n-4}\right)=8 \cdot 2^{k-2}=2 \delta\left(\hat{S}_{n}\right) .
$$

Otherwise $x=1$ and $\beta_{1}$ has to be non-basic by Lemma 2.4 Then $\beta_{1}(1) \geq 2 \delta\left(\hat{S}_{n-4}\right)$ and again $\operatorname{dim} V \geq 8 \delta\left(\hat{S}_{n-4}\right)=2 \delta\left(\hat{S}_{n}\right)$.

Case S6: $H=\hat{S}_{n}, n=2 k$, and $3=p \mid(n-2)$. Set $V^{i}=\operatorname{Ker}\left((c-1)^{i}\right)$ for $i=1,2,3, V^{0}=0$, and $W^{i}=V^{i} / V^{i-1}$. By Lemma 2.3 each $W^{i}$ is a non-zero faithful module for $\hat{S}_{n-3} \leq C_{H}(c)$. Also, at least one of the modules $W^{i}$ is non-basic by Lemma 2.4. Therefore

$$
\operatorname{dim} V \geq 4 \delta\left(\hat{S}_{n-3}\right)=4 \cdot 2^{k-2}=2 \delta\left(\hat{S}_{n}\right) .
$$

Case S7: $H=\hat{S}_{n}, n=2 k+1$, and $3=p \nmid n$. We restrict $V$ to $K:=\left\langle t_{1}\right\rangle \times \hat{A}_{n-3}$ and use the notation of S6. Since $t_{1}$ inverts $c$ and $\hat{A}_{n-3}$ centralizes $c$, each $W^{i}$ is a (non-zero) $K$-module. Let $V \downarrow_{\hat{A}_{n-3}}$ consist of $a+b$ (not necessarily distinct) composition factors, with $a$ of them non-basic and $b$ basic. The argument with $t_{4}$ given in S1 shows that both $a$ and $b$ are even. Also, $a>0$ by Lemma 2.4. Moreover, 
the multiplication by $c-1$ yields injective homomorphisms $W^{3} \hookrightarrow W^{2} \hookrightarrow W^{1}$ as $\hat{A}_{n-3}$-modules, whence $a \geq 3$ or $b \geq 3$. If $a \geq 3$, then $a \geq 4$ as $a$ is even, whence

$$
\operatorname{dim} V \geq 4 \cdot 2 \delta\left(\hat{A}_{n-3}\right)=8 \cdot 2^{k-2}=2 \delta\left(\hat{S}_{n}\right) .
$$

If $a=2$, then $b \geq 4$, whence

$$
\operatorname{dim} V \geq(2 a+b) \delta\left(\hat{A}_{n-3}\right) \geq 8 \delta\left(\hat{A}_{n-3}\right)=2 \delta\left(\hat{S}_{n}\right) .
$$

The induction step for $\hat{S}_{n}$ is complete. So we now assume that the result holds for $\hat{S}_{n}$.

Case A1: $H=\hat{A}_{n}$, and either $p \mid n=2 k$ or $p \nmid n=2 k+1$. Using the result for $\hat{S}_{n}$, we get

$$
\operatorname{dim} V \geq(1 / 2) \cdot 2 \cdot \delta\left(\hat{S}_{n}\right)=2 \delta\left(\hat{A}_{n}\right) .
$$

Case A2: $H=\hat{A}_{n}, n=2 k+1$, and $p \mid n$. Since $V$ is non-basic, at least one of the composition factors of $V \downarrow_{\hat{A}_{n-1}}$ is non-basic. Hence $\operatorname{dim} V \geq 2 \delta\left(\hat{A}_{n-1}\right)=2 \delta\left(\hat{A}_{n}\right)$.

Case A3: $H=\hat{A}_{n}, n=2 k, p \nmid n, p \nmid(n-4)$, and $p \neq 3$. The same argument as in S5 but applied to $K=\hat{A}_{4} * \hat{A}_{n-4}$ yields

$$
\operatorname{dim} V \geq 8 \delta\left(\hat{A}_{n-4}\right)=8 \cdot 2^{k-3}=2 \delta\left(\hat{A}_{n}\right) .
$$

Case $A 4: H=\hat{A}_{n}, n=2 k, p \nmid n, p \nmid(n-3)$, and $p \neq 3$. In this and the next cases we restrict $V$ to the subgroup $G=\hat{A}_{n-3} \cdot\langle u\rangle$ inside $N_{H}(\langle c\rangle)$, where $u=t_{1} t_{4}$. First, we prove:

(*) Let $p \geq 3, n=2 k, p \nmid(n-3)$, and $W$ be a faithful $\mathbb{F} G$-module of dimension less than $2^{k-1}$. Then $W$ is irreducible of dimension $2^{k-2}$ and $W \downarrow_{\hat{A}_{n-3}}$ involves only basic spin modules.

Indeed, let us identify all the groups under consideration with subgroups of $G L(W)$. Let $\mathbf{i} \in G L(W)$ be the multiplication by a primitive fourth root of unity (in $\mathbb{F}$ ) on $W$. Then $(\mathbf{i} u)^{2}=\mathbf{i}^{2} z=1=\left(t_{4}\right)^{2}$. Next, $\mathbf{i}$ and $t_{1}$ centralize $\hat{A}_{n-3}$, so the (conjugation) action of $\mathbf{i} u$ on $\hat{A}_{n-3}$ is exactly the same as that of $t_{4}$. It follows that $B:=\hat{A}_{n-3} \cdot\langle\mathbf{i} u\rangle \cong \hat{S}_{n-3}$. Now $W$ is also a faithful irreducible $B$-module of dimension less than $2^{k-1}=2 \delta\left(\hat{S}_{n-3}\right)$, whence $W$ is basic for $B$, of dimension $2^{k-2}$, and so $W \downarrow_{\hat{A}_{n-3}}$ involves only basic modules.

Abusing the terminology, we will call $W$ as in $(*)$ a basic module for $G$.

As in S3, $V_{0}$ and $V^{\prime}:=V_{1} \oplus V_{2}$ are non-zero faithful $G$-modules. By Lemma 2.4 at least one of $V_{0}$ and $V^{\prime}$ have to involve non-basic $G$-modules. If both of them involve non-basic modules, or only one of them involves a non-basic module and one of them is not irreducible, then by $(*), \operatorname{dim} V \geq 2 \cdot 2^{k-1}=2 \delta\left(\hat{A}_{n}\right)$. Hence we may assume that both of them are irreducible and exactly one of them is non-basic, and that $\operatorname{dim} V<2^{k}$.

Assume first that $V_{0}$ is non-basic and $V^{\prime}$ is basic. Then $\operatorname{dim} V^{\prime} \leq\left(\operatorname{dim} V_{0}\right) / 2$ by $(*)$, and so $\operatorname{dim} V \leq 3\left(\operatorname{dim} V_{0}\right) / 2$. By the definition of $V^{\prime}$, the trace of $c$ on $V^{\prime}$ is $-\left(\operatorname{dim} V^{\prime}\right) / 2$. Hence

$$
\varphi(c)=\operatorname{dim} V_{0}-\left(\operatorname{dim} V^{\prime}\right) / 2 \geq 3\left(\operatorname{dim} V_{0}\right) / 4 \geq(\operatorname{dim} V) / 2=\varphi(1) / 2,
$$

contrary to Lemma 2.5.

Assume next that $V_{0}$ is basic, with Brauer $G$-character $\varphi_{0}$, and $V^{\prime}$ is non-basic, with Brauer $G$-character $\varphi^{\prime}$. Then

$$
\varphi(c)=\operatorname{dim} V_{0}-\left(\operatorname{dim} V^{\prime}\right) / 2=2^{k-2}-\varphi^{\prime}(1) / 2 .
$$


Choosing a conjugate $c^{\prime}$ of $c$ lying in $\hat{A}_{n-3}$ we have

$$
\varphi\left(c^{\prime}\right)=\varphi_{0}\left(c^{\prime}\right)+\varphi^{\prime}\left(c^{\prime}\right)=-2^{k-3}+\varphi^{\prime}\left(c^{\prime}\right) .
$$

Hence

$$
\varphi^{\prime}\left(c^{\prime}\right)=3 \cdot 2^{k-3}-\varphi^{\prime}(1) / 2>0,
$$

as $\varphi^{\prime}(1)=\operatorname{dim} V-\operatorname{dim} V_{0}<3 \cdot 2^{k-2}$ by our assumption. Next, we look at the element $d=c c^{\prime}$. Decompose $V^{\prime}$ as the sum of two non-trivial $c$-eigenspaces and let $\alpha, \beta$ be the characters of $\hat{A}_{n-3}$ on them. Since $t_{1}$ inverts $c$ and centralizes $\hat{A}_{n-3}$, we have $\beta(g)=\alpha\left(t_{1} g t_{1}\right)=\alpha(g)$ for any $g \in \hat{A}_{n-3}$. Thus

$$
\varphi^{\prime}(d)=\omega \alpha\left(c^{\prime}\right)+\omega^{2} \beta\left(c^{\prime}\right)=\left(\omega+\omega^{2}\right) \alpha\left(c^{\prime}\right)=-\alpha\left(c^{\prime}\right)=-\varphi^{\prime}\left(c^{\prime}\right) / 2 .
$$

Using (3.2), the assumption that $V_{0}$ is basic, and $\operatorname{dim} V<2^{k}$, we now obtain

$$
\begin{aligned}
\varphi(d) & =\varphi_{0}(d)+\varphi^{\prime}(d)=\varphi_{0}\left(c^{\prime}\right)-\varphi^{\prime}\left(c^{\prime}\right) / 2 \\
& =-\varphi_{0}(1) / 2-\varphi^{\prime}\left(c^{\prime}\right) / 2<-2^{k-3}<-\varphi(1) / 8,
\end{aligned}
$$

contrary to Lemma 2.5.

Case A5: $H=\hat{A}_{n}, n=2 k, p \nmid n, p \nmid(n-3)$, and $p=3$. We use the notation of A4 and S6. Arguing as in S6, we see that each $W^{i}$ is a non-zero faithful $G$-module, and by Lemma 2.4, at least one of them is non-basic. According to the claim proved in $\mathrm{A} 5, \operatorname{dim} V \geq 2^{k-1}+2 \cdot 2^{k-2}=2 \delta\left(\hat{A}_{n}\right)$, as desired.

The proof of Theorem A is complete.

The following easy corollary of Theorem A will often be used in what follows.

Corollary 3.1. Let $n \geq 5, H=\hat{S}_{n}$ or $\hat{A}_{n}$, and $D$ be an irreducible faithful $\mathbb{F} H$ module. If $G$ is a subgroup of $H$ containing $Z(H)$ and such that $D \downarrow_{G}$ is irreducible, then

$$
|\pi(G)| \geq(\operatorname{dim} D)^{2} \geq 2^{(n-4)} .
$$

Proof. Indeed, the second inequality comes from Theorem A if $n \geq 8$ and [12, 19. if $n=5,6,7$. As $D \downarrow_{G}$ is irreducible and $D$ is faithful, $G$ has a faithful irreducible complex representation of degree $\geq \operatorname{dim} D$. The sum of squared degrees of irreducible complex representations of $G$ that are trivial on $Z$ equals $|\pi(G)|$. Hence $|G|-|\pi(G)|=|\pi(G)|$ is at least $(\operatorname{dim} D)^{2}$.

\section{BASIC SPIN MODULES}

Basic modules play a very special role in the representation theory of $\hat{S}_{n}$ and $\hat{A}_{n}$. In this section we will study restrictions of these modules to subgroups $G$ such that $\pi(G)$ is either a Young subgroup or a wreath product subgroup. These subgroups are important because among them we find maximal imprimitive subgroups.

We will have to use the terminology of superalgebra and some result from [8, 9. We review what is needed, referring the reader to [8, 9] for details. Let $\mathcal{S}_{n}$ be the twisted group algebra of $S_{n}$. This may be described as the superalgebra with degree $\overline{1}$ generators $t_{1}, \ldots, t_{n-1}$ and relations $t_{i}^{2}=1, t_{i} t_{j}=-t_{j} t_{i}, t_{i} t_{i+1} t_{i}=t_{i+1} t_{i} t_{i+1}$ for all admissible $i, j$ with $|i-j|>1$. Any subgroup $H$ of $S_{n}$ yields a sub(super)algebra $\mathcal{H} \subset \mathcal{S}_{n}$. We identify spin modules over $\hat{S}_{n}$ with modules over $\mathcal{S}_{n}$ (there is an isomorphism of categories) and do the same for $\hat{H}$ and $\mathcal{H}$. Thus we are interested in irreducible restrictions from $\mathcal{S}_{n}$ to $\mathcal{H}$. If $H$ is a standard Young subgroup $S_{a_{1}} \times$ $\cdots \times S_{a_{b}}<S_{n}$, then we have $\mathcal{H} \cong \mathcal{S}_{a_{1}} \otimes \cdots \otimes \mathcal{S}_{a_{b}}$ where the tensor product is the 
tensor product of superalgebras, i.e. to multiply the tensors we use the usual sign rule.

We recall also that irreducible supermodules over superalgebras can be of two types: type $\mathrm{M}$ when the supermodule is irreducible considered as a usual module, and type $\mathrm{Q}$ when the supermodule splits as a direct sum of two non-isomorphic irreducible modules considered as a usual module. If $A$ and $B$ are superalgebras, $V$ is an $A$-supermodule, and $W$ is a $B$-supermodule, we denote by $V \otimes W$ the outer tensor product of $V$ and $W$, which is an $A \otimes B$-supermodule. Note that we use the sign rule to define the action of $A \otimes B$ on $V \otimes W$, namely

$$
(a \otimes b)(v \otimes w)=(-1)^{\operatorname{deg} b \operatorname{deg} v} a v \otimes b w
$$

for homogeneous elements $a \in A, b \in B, v \in V, w \in W$. If $V$ and $W$ are both irreducible of type $\mathrm{M}$, then $V \otimes W$ is also irreducible of type M. If one of $V$ and $W$ is of type $\mathrm{M}$ and another one is of type Q, then $V \otimes W$ is irreducible of type $\mathbf{Q}$. Finally if both $V$ and $W$ are irreducible of type $Q$, then $V \otimes W$ splits as a direct sum of two copies of the same irreducible supermodule of type M, which is denoted by $V \circledast W$. To have a unified notation we also denote $V \otimes W$ by $V \circledast W$ in the first two cases. Thus, $\circledast$ is an operation which always takes a pair of irreducible supermodules to an irreducible supermodule.

An example of a superalgebra is obtained as follows. Let $G$ be a finite group and $H<G$ be a subgroup of index two. Let $\mathcal{A}$ be a group algebra of $G$ with canonical basis $\left\{t_{g} \mid g \in G\right\}$. Define the $\mathbb{Z}_{2}$-grading on $\mathcal{A}$ by setting $\mathcal{A}_{\overline{0}}:=\operatorname{span}\left\{t_{g} \mid g \in H\right\}$ and $\mathcal{A}_{\overline{1}}:=\operatorname{span}\left\{t_{g} \mid g \notin H\right\}$. Let sgn denote the 1-dimensional non-trivial $\mathbb{F} G$ module with trivial action of $H$. Let $L_{1}^{ \pm}, \ldots, L_{k}^{ \pm}, L_{k+1}, \ldots, L_{m}$ be a complete list of irreducible non-isomorphic $\mathbb{F} G$-modules, such that $L_{i}^{ \pm} \otimes \mathbf{s g n} \cong L_{i}^{\mp}$ for $1 \leq i \leq k$ and $L_{i} \otimes \mathbf{s g n} \cong L_{i}$ for $k<i \leq m$. An application of the Clifford theory implies that there are $m$ irreducible $\mathcal{A}$-supermodules $\mathcal{L}_{1}, \ldots, \mathcal{L}_{m}$ such that, considered as usual modules, $\mathcal{L}_{i}=L_{i}^{+} \oplus L_{i}^{-}$for $1 \leq i \leq k$ and $\mathcal{L}_{i}=L_{i}$ for $k<i \leq m$, and these are all irreducible $\mathcal{A}$-supermodules up to isomorphism.

Recall the basic modules $Y_{n}^{( \pm)}$from Lemma 2.1 By general facts described in the previous paragraph and Lemma 2.1 there is an irreducible supermodule $\mathcal{Y}_{n}$, called the basic supermodule, which is determined uniquely up to an isomorphism by the following property. Considered as a usual module, $\mathcal{Y}_{n}$ is $Y_{n}$ if $n$ is even and $p \mid n$ or $n$ is odd and $p \nmid n$, and it is $Y_{n}^{+} \oplus Y_{n}^{-}$otherwise; see Lemma 2.1. Correspondingly, the supermodule $\mathcal{Y}_{n}$ is of type $\mathrm{M}$ if and only if $n$ is even and $p \mid n$, or $n$ is odd and $p \nmid n$. By Lemma 2.1, we have

$$
\operatorname{dim} \mathcal{Y}_{n}= \begin{cases}2^{n / 2} & \text { if } n \text { is even and } p \not \mid n \\ 2^{(n-1) / 2} & \text { if } n \text { is odd, } \\ 2^{(n-2) / 2} & \text { if } n \text { is even and } p \mid n .\end{cases}
$$

Similarly, if the ground field is $\mathbb{C}$, we get the basic spin supermodule $\mathcal{Y}_{n, \mathbb{C}}$ which is of type $\mathrm{M}$ if and only if $n$ is odd. We have

$$
\operatorname{dim} \mathcal{Y}_{n, \mathbb{C}}= \begin{cases}2^{n / 2} & \text { if } n \text { is even } \\ 2^{(n-1) / 2} & \text { if } n \text { is odd }\end{cases}
$$

Lemma 4.1. Let $\lambda=\left(a_{1}, a_{2}, \ldots, a_{b}\right)$ be a composition of $n$, and

$$
\hat{S}_{\lambda}=\pi^{-1}\left(S_{a_{1}} \times S_{a_{2}} \times \cdots \times S_{a_{b}}\right)<\hat{S}_{n}, \quad \hat{A}_{\lambda}=\hat{A}_{n} \cap S_{\lambda} .
$$


Suppose that $V$ (resp. $\left.V^{\prime}\right)$ is an irreducible $\mathbb{F} \hat{S}_{\lambda^{-}}\left(\right.$resp. $\left.\mathbb{F} \hat{A}_{\lambda^{-}}\right)$module such that composition factors of every restriction $V \downarrow_{\hat{S}_{a_{i}}}$ (resp. $V^{\prime} \downarrow_{\hat{A}_{a_{i}}}$ ), $i=1,2, \ldots, b$, are basic spin. Then

$\operatorname{dim} V=2^{(n-s-u) / 2-t-\lfloor(r+s+1) / 2\rfloor} \quad\left(\right.$ resp. $\left.\operatorname{dim} V^{\prime}=2^{(n-s-u) / 2-t-\lfloor(r+s+2) / 2\rfloor}\right)$, where

$$
\begin{aligned}
r & =\sharp\left\{i \mid a_{i} \text { is even and } p \nmid a_{i}\right\}, \\
s & =\sharp\left\{i \mid a_{i} \text { is odd and } p \mid a_{i}\right\}, \\
t & =\sharp\left\{i \mid a_{i} \text { is even and } p \mid a_{i}\right\}, \\
u & =\sharp\left\{i \mid a_{i} \text { is odd and } p \nmid a_{i}\right\} .
\end{aligned}
$$

Proof. Let $\mathcal{V}$ be an irreducible supermodule over $\mathcal{S}\left(a_{1}\right) \otimes \cdots \otimes \mathcal{S}\left(a_{b}\right)$ such that all composition factors of its restriction to any $\mathcal{S}\left(a_{i}\right)$ are basic. Then $\mathcal{V}=$ $\mathcal{Y}_{a_{1}} \circledast \cdots \circledast \mathcal{Y}_{a_{b}}$. Moreover, $\operatorname{dim} V=\operatorname{dim} V^{\prime}=(\operatorname{dim} \mathcal{V}) / 2$ if $\mathcal{V}$ is of type $\mathbf{Q}$ and $\operatorname{dim} V=2 \operatorname{dim} V^{\prime}=\operatorname{dim} \mathcal{V}$ if $\mathcal{V}$ is of type M. Therefore

$$
\operatorname{dim} V=2^{-\lfloor(r+s+1) / 2\rfloor} \prod_{i=1}^{b} \operatorname{dim} \mathcal{Y}_{a_{i}}, \quad \operatorname{dim} V^{\prime}=2^{-\lfloor(r+s+2) / 2\rfloor} \prod_{i=1}^{b} \operatorname{dim} \mathcal{Y}_{a_{i}},
$$

which implies the result.

Corollary 4.2. Let $H=\hat{S}_{n}$ or $\hat{A}_{n}, D$ be a basic spin $\mathbb{F} H$-module, and $\lambda=$ $\left(\lambda_{1}, \ldots, \lambda_{h}\right)$ be a composition of $n$ with $h>1$ non-zero parts. Let $G$ be $\hat{S}_{\lambda}$ or $\hat{A}_{\lambda}$. Then the restriction $D \downarrow_{G}$ is irreducible if and only if $p \nmid \lambda_{i}$ for all $i=1,2, \ldots, h$ and one of the following happens:

(i) $H=\hat{S}_{n}, G=\hat{S}_{\lambda}$, and either $n$ is even and $h=2$, or $n$ is odd, $p \mid n$, and $h \leq 3$.

(ii) $H=\hat{S}_{n}, G=\hat{A}_{\lambda}, n$ is odd, $p \mid n$, and $h=2$.

(iii) $H=\hat{A}_{n}, G=\hat{A}_{\lambda}$, and either $n$ is odd and $h=2$, or $n$ is even, $p \mid n$, and $h \leq 3$.

Theorem 4.3. Let $D$ be a basic spin $\mathbb{F} \hat{S}_{n}$-module, and let $G$ be a maximal subgroup of $\hat{S}_{n}$ with $\pi(G)$ imprimitive. Then $D \downarrow_{G}$ is irreducible if and only if one of the following holds:

(i) $\pi(G)=S_{n-a} \times S_{a}, a<n / 2, p \nmid a, p \nmid(n-a)$, and either $n$ is even, or $n$ is odd and $p \mid n$.

(ii) $\pi(G)=S_{a} \prec S_{b}$ for some $a, b \geq 2$ with $n=a b$ and $p \nmid a$.

Proof. By assumption we only need to consider subgroups $G$ with $\pi(G)=S_{n-a} \times$ $S_{a}, a<n / 2$, or $\pi(G)=S_{a} \nmid S_{b}, a, b \geq 2, a b=n$. In the former case the result follows from Corollary 4.2. So let $\pi(G)=S_{a}$ 々 $S_{b}$. We will sometimes write $G_{a, b}$ for G. Denote

$$
K_{a, b}:=\pi^{-1}\left(S_{a} \times \cdots \times S_{a}\right) \triangleleft G .
$$

Then $G_{a, b} / K_{a, b} \cong S_{b}$. We will also need the subgroup

$$
B:=\pi^{-1}\left(S_{a} \curlywedge A_{b}\right) \triangleleft G_{a, b} .
$$

It is proved in [22] that the restriction to $G$ of a basic module $D_{\mathbb{C}}$ over the field of complex numbers to $G$ is irreducible.

Consider the irreducible $\mathbb{F} K_{a, b}$-supermodule $\mathcal{Y}_{a} \circledast \cdots \circledast \mathcal{Y}_{a}$, where $\mathcal{Y}_{a}$ appears $b$ times. This supermodule is of type $\mathbf{Q}$ if and only if $b$ is odd and either $a$ is even and 
$p \nmid a$, or $a$ is odd and $p \mid a$. Now consider $\mathcal{Y}_{a} \circledast \cdots \circledast \mathcal{Y}_{a}$ as a usual module. Then it has two composition factors, $M_{a, b}^{+}$and $M_{a, b}^{-}$say, when the supermodule is of type Q, and only one composition factor, $M_{a, b}$ say, otherwise. The dimensions of $M_{a, b}^{( \pm)}$ can be found using Lemma 4.1 Note that $M_{a, b}^{( \pm)}$are the only possible composition factors of the restriction $D \downarrow_{K_{a, b}}$.

Now we apply Clifford theory to identify possible composition factors of the restriction $D \downarrow_{G}$. From the previous paragraph, we conclude that these factors may be of two forms:

(a) $V \otimes W$, where $V$ and $W$ are (possibly projective) irreducible representations of $\mathbb{F} G$ such that $V \downarrow_{K_{a, b}} \cong M_{a, b}^{( \pm)}$and $K_{a, b}$ acts trivially on $W$. Here the inertia group is $G$.

(b) $\operatorname{ind}_{B}^{G}(V \otimes W)$, where $V$ and $W$ are (possibly projective) irreducible $\mathbb{F} B$ representations such that $V \downarrow_{K_{a, b}} \cong M_{a, b}^{ \pm}$and $K_{a, b}$ acts trivially on $W$. Here the inertia group is $B$.

In case (a) (resp. (b)), $W$ may be a considered as a (possibly projective) representation of $\mathbb{F} S_{b}$ (resp. $\mathbb{F} A_{b}$ ). We claim that this representation must be basic spin. By a reduction modulo $p$ argument it suffices to prove this over $\mathbb{C}$. As $D_{\mathbb{C}} \downarrow_{G}$ is irreducible, it must be isomorphic to a module described in (a) or (b), namely $V_{\mathbb{C}} \otimes W_{\mathbb{C}}$ or $\operatorname{ind}_{B}^{G}\left(V_{\mathbb{C}} \otimes W_{\mathbb{C}}\right)$ with $V_{\mathbb{C}} \downarrow_{K_{a, b}} \cong M_{a, b, \mathbb{C}}^{( \pm)}$. Using known dimensions of $D_{\mathbb{C}}$ and $M_{a, b, \mathbb{C}}^{( \pm)}$, we find that $\operatorname{dim} W_{\mathbb{C}}$ is equal to the dimension of the basic module $Y_{b, \mathbb{C}}^{( \pm)}\left(\right.$resp. $\left.U_{b, \mathbb{C}}^{( \pm)}\right)$if the restriction is of type (a) (resp. type (b)). Using [2, 2.4], we deduce that $W_{\mathbb{C}}$ is not a linear representation if $b \geq 10$. In this case Theorem $\mathrm{A}$ implies that $W_{\mathbb{C}}$ is basic spin. Finally, for small values of $b$, pick sufficiently large $c>b$, and note that composition factors of $Y_{a b, \mathbb{C}}^{( \pm)} \downarrow_{G_{a, b}}$ are among the composition factors of the restrictions $Y_{a c, \mathbb{C}}^{( \pm)} \downarrow_{G_{a, c}} \downarrow_{G_{a, b}}$. Now the required result follows from the one for $c \geq 10$, as all composition factors of the restriction $Y_{c, \mathbb{C}}^{( \pm)} \downarrow_{\hat{S}_{b}}$ are basic spin.

We also note that the restriction $Y_{n, \mathbb{C}}^{( \pm)} \downarrow_{G}$ is of type (b) if and only if $a$ is even and $b$ is odd. Indeed, in all other cases there is only one irreducible $\mathbb{C} K_{a, b}$-module $M_{a, b, \mathbb{C}}$, which can appear as a composition factor, so $Y_{n, \mathbb{C}}^{( \pm)} \downarrow_{G}$ could only be of type (a). On the other hand, if $a$ is even and $b$ is odd, there are two possible composition factors $M_{a, b, \mathbb{C}}^{+}$and $M_{a, b, \mathbb{C}}^{-}$. To see that in this case the restrictions $Y_{n, \mathbb{C}}^{ \pm} \downarrow_{G}$ are of type (b), it suffices to note that $Y_{n, \mathbb{C}}^{ \pm} \downarrow_{K_{a, b}} \cong M_{a, b, \mathbb{C}}^{+} \oplus M_{a, b, \mathbb{C}}^{-}$. If the last equality does not hold, then (up to a choice of signs) we have $Y_{n, \mathbb{C}}^{ \pm} \downarrow_{K_{a, b}} \cong 2 M_{a, b, \mathbb{C}}^{ \pm}$, and in particular $Y_{n, \mathbb{C}}^{+} \downarrow_{K_{a, b}} \neq Y_{n, \mathbb{C}}^{-} \downarrow_{K_{a, b}}$. But the characters of $Y_{n, \mathbb{C}}^{+}$and $Y_{n, \mathbb{C}}^{-}$only differ on conjugacy classes corresponding to the $n$-cycle [22, 2A], and there are no such elements in $K_{a, b}$, which shows that the characters of the restrictions $Y_{n, \mathbb{C}}^{+} \downarrow_{K_{a, b}}$ and $Y_{n, \mathbb{C}}^{-} \downarrow_{K_{a, b}}$ are the same, giving a contradiction.

Now we consider various cases.

Case 1. $a$ is odd and $p \nmid\left\{\right.$. Here $\operatorname{dim} M_{a, b}=2^{(a b-b) / 2}$, and all composition factors of the restriction $D \downarrow_{G}$ must be of type (a) above. Now one checks that in all cases we have $\operatorname{dim} D=\operatorname{dim} M_{a, b} \operatorname{dim} Y_{b}^{( \pm)}$, so $D \downarrow_{G}$ must be irreducible.

Case 2. $a$ is odd and $p \mid a$. If $b$ is even, then we have $\operatorname{dim} M_{a, b}=2^{(a b-2 b) / 2}$, and all composition factors of the restriction $D \downarrow_{G}$ must be of type (a) above. Now one checks that $\operatorname{dim} D>\operatorname{dim} M_{a, b} \operatorname{dim} Y_{b}^{( \pm)}$, so $D \downarrow_{G}$ could not be irreducible. If $b$ is 
odd, then we have $\operatorname{dim} M_{a, b}^{ \pm}=2^{(a b-2 b-1) / 2}$, and so whether we have a composition factor of type (a) or (b), its dimension is strictly less than that of $D$, and so the restriction is reducible again.

Case 3. $a$ is even and $p \mid a$. We have $\operatorname{dim} M_{a, b}=2^{(a b-2 b) / 2}$, and we can use dimensions as in Case 2 to see that the restriction is reducible.

Case 4. $a$ is even, $p \nmid\left\{a, b\right.$ is even. We have $\operatorname{dim} M_{a, b}=2^{(a b-b) / 2}$, and the restriction is irreducible by dimensions.

Case 5. $a$ is even, $p \nmid\left\{a, b\right.$ is odd. We have $\operatorname{dim} M_{a, b}^{ \pm}=2^{(a b-b-1) / 2}$. Assume first that $p \nmid b$. Then $\operatorname{dim} Y_{b}=2^{(b-1) / 2}$, and $\operatorname{dim} U_{b}^{ \pm}=2^{(b-3) / 2}$; see Lemma 2.1 Now a composition factor of $D \downarrow_{G}$ of type (a) has dimension

$$
2^{(a b-b-1) / 2} \cdot 2^{(b-1) / 2}=2^{(n-2) / 2}=\operatorname{dim} D,
$$

so $D \downarrow_{G}$ is irreducible if such a composition factor occurs. On the other hand, a composition factor of type (b) has dimension $2 \cdot 2^{(a b-b-1) / 2} \cdot 2^{(b-3) / 2}=\operatorname{dim} D$, so $D \downarrow_{G}$ is irreducible anyway. We finally assume that $p \mid b$. Then $\operatorname{dim} Y_{b}^{ \pm}=\operatorname{dim} U_{b}=2^{(b-3) / 2}$, and the dimension argument as above shows that the restriction is irreducible if there is a composition factor of type (b), but it is reducible otherwise. So in this final case we do have to figure out what composition factors occur. We proved above that $D_{\mathbb{C}} \downarrow_{G} \cong \operatorname{ind}_{B}^{G}\left(V_{\mathbb{C}} \otimes U_{b, \mathbb{C}}^{ \pm}\right)$. Now, we reduce both sides of this equality modulo $p$ to see that composition factors of the restriction $D \downarrow_{G}$ are of type (b), which completes the proof.

Theorem 4.4. Let $D$ be a basic spin $\mathbb{F} \hat{A}_{n}$-module, and let $H$ be a maximal subgroup of $\hat{A}_{n}$ with $\pi(H)$ imprimitive. Then $D \downarrow_{H}$ is irreducible if and only if one of the following holds:

(i) $\pi(H)=A_{n} \cap\left(S_{n-a} \times S_{a}\right), a<n / 2, p \nmid a, p \nmid(n-a)$, and either $n$ is odd, or $n$ is even and $p \mid n$.

(ii) $\pi(H)=A_{n} \cap\left(S_{a} \backslash S_{b}\right)$ for some $a, b \geq 2$ with $n=a b$ and $p \nmid a$.

Proof. (i) is proved using Corollary 4.2. We now prove (ii). Let $G=\pi^{-1}\left(S_{a} S_{b}\right)$. Then $H$ is a subgroup of $G$ of index 2 . We will use notation introduced in the proof of Theorem 4.3.

Assume first that there is only one basic spin module $Y_{n}$ over $\hat{S}_{n}$, and the restriction $Y_{n} \downarrow_{G}$ is irreducible. Then the module

$$
U_{n}^{+} \downarrow_{H} \oplus U_{n}^{-} \downarrow_{H} \cong Y_{n} \downarrow_{G} \downarrow_{H}
$$

has at most two composition factors. This proves that $U_{n}^{ \pm} \downarrow_{H}$ is irreducible. Thus $D \downarrow_{H}$ is irreducible in the cases where either $n$ is odd and $p \nmid n$ or $n$ is even, $p \nmid a$, and $p \mid b$. Now we consider the remaining cases.

Case 1. $n$ is even and $p \nmid \not n$. In this case $p \nmid \backslash a$ and $p \nmid \not b$, so reductions modulo $p$ of (the complex analogues of) all the modules involved are irreducible, and the result follows from the corresponding result over $\mathbb{C}$; see 22$]$.

Case 2. $n$ is odd and $p \mid n$. Assume first that $p \mid a$. Composition factors of $U_{n} \downarrow_{H}$ are restrictions from $G$ to $H$ of composition factors of $Y_{n}^{ \pm} \downarrow_{G}$, whose dimension is at most $2^{(a b-b-2) / 2}$ (see the proof of Theorem 4.3), while $\operatorname{dim} U_{n}=2^{(a b-3) / 2}$. Therefore $U_{n} \downarrow_{H}$ must be reducible. Now, let $p \nmid a$. By Lemma2.1 we have $Y_{n}^{ \pm} \downarrow_{\hat{A}_{n}}=$ $U_{n}$, and by Theorem 4.3, the restriction $Y_{n}^{ \pm} \downarrow_{G}$ is irreducible. We have $Y_{n, \mathbb{C}} \downarrow_{G}=$ $V_{\mathbb{C}} \otimes Y_{b, \mathbb{C}}$. Reducing modulo $p$ we get $\left(Y_{n}^{+}+Y_{n}^{-}\right) \downarrow_{G}=V \otimes\left(Y_{b}^{+}+Y_{b}^{-}\right)$, so up to a choice of signs we must have $Y_{n}^{ \pm} \downarrow_{G}=V \otimes Y_{b}^{ \pm}$. By uniqueness in Clifford theory, 
we have $V \otimes Y_{b}^{+} \not V \otimes Y_{b}^{-}$. It follows that $V \otimes Y_{b}^{+} \cong\left(V \otimes Y_{b}^{-}\right) \otimes$ sgn, so the restrictions $V \otimes Y_{b}^{ \pm} \downarrow_{H}$ are irreducible, whence $D \downarrow_{H}$ is also irreducible.

Case 3. $a$ is even, $b$ is odd, and $p \mid a$. We introduce the new subgroup

$$
K_{a, b}^{\prime}:=K_{a, b} \cap \hat{A}_{n} .
$$

Note that $K_{a, b}^{\prime} \triangleleft H$, and $H / K_{a, b}^{\prime} \cong S_{b}$. We apply Clifford theory to this situation. By Lemma 4.1 $\operatorname{dim} M_{a, b}=2^{(a b-2 b) / 2}$. Also, $M_{a, b}$ comes from the corresponding supermodule of type $\mathrm{M}$, so on restriction to $K_{a, b}^{\prime}$ it splits as a direct sum of two irreducible modules of dimension $2^{(a b-2 b-2) / 2}$. If the inertia group of a composition factor $W$ of $D \downarrow_{H}$ is $H$, then we get from Clifford theory that

$$
\operatorname{dim} W=2^{(a b-2 b-2) / 2} \operatorname{dim} Y_{b}<2^{(a b-4) / 2}=\operatorname{dim} D,
$$

so the restriction $D \downarrow_{H}$ is reducible. Otherwise the inertia group is $K_{a, b}^{\prime} A_{b}<H$, in which case we still have

$$
\operatorname{dim} W=2 \cdot 2^{(a b-2 b-2) / 2} \operatorname{dim} U_{b}^{( \pm)}=2^{(a b-b-3) / 2}<\operatorname{dim} D
$$

as $b \geq 3$

Case 4. $b$ is even, and $p \mid a$. Here $\operatorname{dim} U_{n}^{ \pm}=2^{(a b-4) / 2}$, and composition factors of $Y_{n} \downarrow_{G}$ have dimensions $2^{(a b-b-2) / 2}$, so we may assume that $b=2$. Set

$$
J:=\pi^{-1}\left(A_{a} \times A_{a}\right) .
$$

Also, pick an element $y$ in $\pi^{-1}((1, a+1)(2, a+2) \ldots(a, 2 a))$ if $a$ is even and $\pi^{-1}((1, a+1)(2, a+2) \ldots(a, 2 a)(1,2))$ if $a$ is odd. Finally, set $x=t_{1} t_{a}$ where $t_{i}$ are the standard generators of $\hat{S}_{n}$. Then $J \triangleleft H, K^{\prime}:=K_{a, 2}^{\prime}=\langle J, x\rangle$, and $H=\langle J, x, y\rangle$.

Assume now that $a$ is odd. Then $\pi\left(y^{2}\right)=\pi(x)$, whence $H / J \cong \mathbb{Z}_{4}$. Let $W$ be any composition factor of $D \downarrow_{H}$. Every composition factor of $W \downarrow_{J}$ must be isomorphic to the module $M:=U_{a} \otimes U_{a}$ of dimension $2^{a-3}$, so $M$ must be $H$-stable. Since $H / J$ is cyclic, $M$ extends to $H$, so $\operatorname{dim} W=2^{a-3}<2^{a-2}=\operatorname{dim} D$.

Finally, let $a$ be even. Now, there are two basic modules $U_{a}^{ \pm}$for $\hat{A}_{a}$, and $\operatorname{dim} U_{a}^{ \pm}=2^{(a-4) / 2}$. It follows that there can be two different composition factors $V_{1}$ and $V_{2}$ in the restriction $D \downarrow_{K^{\prime}}$, characterized by $V_{1} \downarrow_{J}=U_{a}^{+} \otimes U_{a}^{+} \oplus U_{a}^{-} \otimes U_{a}^{-}$ and $V_{2} \downarrow_{J}=U_{a}^{+} \otimes U_{a}^{-} \oplus U_{a}^{-} \otimes U_{a}^{+}$. Observe that the Brauer characters of $V_{1}$ and $V_{2}$ are zero on $K^{\prime} \backslash J$ and y stabilizes $V_{1} \downarrow J$ and $V_{2} \downarrow J$. It follows that $y$ stabilizes the Brauer characters of $V_{1}$ and $V_{2}$, so $V_{1}$ and $V_{2}$ extend to $H$, whence the dimension of every composition factor of $D \downarrow_{H}$ equals $\operatorname{dim} V_{i}$, which is less than $\operatorname{dim} D$. Thus in both subcases $D \downarrow_{H}$ is reducible.

\section{Primitive subgroups (Theorem B)}

In this section we prove Theorem B. Assume $D \downarrow_{G}$ is irreducible. Without loss of generality we may assume that $G$ contains $Z:=Z(H)$ (however, $Z$ may split out in $G$ ). The cases $n=5,6,7$ can be checked easily using [12] and [19], so we will assume that $n \geq 8$.

In view of Corollary [3.1, we may assume that $\pi(G)$ is a primitive subgroup of $S_{n}$ of order $\geq 2^{n-4}$. Such subgroups are classified in [22, 6.2]. We will proceed case by case according to that classification. If $\pi(G)$ is almost simple, we set $S:=\operatorname{soc}(\pi(G))$, and let $\varphi$ be the Brauer character of $D$. We will use results from [12, 19] without special reference. 
A. First we assume that the $\mathbb{F} H$-module $D$ does not lift to characteristic 0 ; that is, it is not a reduction modulo $p$ of an ordinary irreducible module. In particular, $p$ divides $|H|$.

Case $n=8$. Here we have either (a) $S=L_{2}(7)$ or (b) $\mathbb{Z}_{2}^{3} \triangleleft \pi(G) \leq A G L_{3}(2)$. By Lemma 2.2 8 divides $\operatorname{dim} D$. In the case (a) $G$ has no irreducible characters of degree $>8$, so $\operatorname{dim} D=8$. But in this case $D$ lifts to characteristic 0 , a contradiction. In the case (b), using Corollary 3.1, we conclude that $\operatorname{dim} D \leq 32$. But $D$ does not lift to characteristic 0 , so either $p=5$ and $\operatorname{dim} D=32$, or $p=7$ and $\operatorname{dim} D=16$. In particular $|\pi(G)| \geq 16^{2}$, whence $\pi(G)=A G L_{3}(2)=\mathbb{Z}_{2}^{3}: G L_{3}(2)$. As $A:=G L_{3}(2)$ acts irreducibly on $\mathbb{Z}_{2}^{3}$, we have that $K:=\pi^{-1}\left(\mathbb{Z}_{2}^{3}\right) \cong \mathbb{Z}_{2}^{4}$. If $p=5$, then one can view $D \downarrow_{G}$ as an irreducible complex representation of $G$, whence $(\operatorname{dim} D) \mid[G: K]=168$ by Ito's Theorem [16, 6.15], a contradiction. So $p=7$ and $\operatorname{dim} D=16$. Decompose $\varphi \downarrow_{K}=s \sum_{i=1}^{t} \lambda_{i}$, where $t \geq 2$ and $\left\{\lambda_{1}, \ldots, \lambda_{t}\right\}$ is an $A$-orbit on the set of 8 linear characters of $K$ that are faithful on $Z$. As $t \mid \operatorname{dim} D, t$ is a 2-power. Inspecting the subgroups of $A$ we see that $A_{0}:=\operatorname{Stab}_{A}\left(\lambda_{1}\right)$ is isomorphic to $\mathbb{Z}_{7}: \mathbb{Z}_{3}$ and $t=8$. Thus $A$ acts transitively on the 8 linear characters of $K$ that are faithful on $Z$. Now $\varphi \downarrow_{G}=\theta \uparrow{ }^{G}$, where $\theta$ is an irreducible Brauer character of degree 2 of $G_{0}:=K: A_{0}$ and $\theta \downarrow_{K}=2 \lambda_{1}$. The quotient of $G_{0}$ by $\operatorname{Ker}\left(\lambda_{1}\right)$ is $\left(\mathbb{Z}_{2} \times \mathbb{Z}_{7}\right): \mathbb{Z}_{3}$. But $p=7$ so $\mathbb{Z}_{7}$ acts trivially. Thus $\theta$ yields an irreducible character of degree 2 of $\mathbb{Z}_{2}: \mathbb{Z}_{3} \cong \mathbb{Z}_{6}$, a contradiction.

Case $n=9$. Here either (a) $S=L_{2}$ (8) or (b) $\mathbb{Z}_{3}^{2} \triangleleft \pi(G) \leq A G L_{2}(3)$. As $D$ does not lift to characteristic 0 , either $\operatorname{dim} D \geq 48$ or $p=3, H=\hat{S}_{9}$, and $\operatorname{dim} D=8$. But the former case is impossible by Corollary 3.1. In the latter case we must be in (b), as in (a) $G$ has no irreducible 3-Brauer character of degree 8. But then $G$ has a normal subgroup $\mathbb{Z}_{3}^{2}$, which must act trivially on $D$. So $D$ factors through to give an irreducible module of dimension 8 over $G / \mathbb{Z}_{3}^{2}$, which is impossible by Corollary 3.1 .

Case $n=10$. Here $S=A_{5}$ or $S=A_{6}$. As $|\operatorname{Out}(S)| \leq 4$ and $\pi^{-1}(S)$ has no irreducible character of degree $>10$, we have $\operatorname{dim} D \leq 40$. On the other hand, $8 \mid \operatorname{dim} D$ by Lemma 2.2 and $D$ does not lift to characteristic 0 . Hence $p=5$, $H=\hat{A}_{10}$ and $\operatorname{dim} D=8$. Then $S \neq A_{5}$. The analysis in [22. p. 463] shows that $\pi(G)=A_{6}$ or $M_{10}$ and $\pi^{-1}(S)=\mathbb{Z}_{2} \times A_{6}$. Now one checks that $D \downarrow_{\pi^{-1}(S)}$ is in fact irreducible, giving exceptions in (ii)(g) and (ii)(f).

Case $n=11$. Here $S$ is $L_{2}(11)$ or $M_{11}$. Checking the irreducible characters of $G$, we conclude that $\operatorname{dim} D \leq 55$. Also, $D$ does not lift to characteristic 0 . Therefore $p=11, H=\hat{S}_{11}, \operatorname{dim} D=16$, and $S=M_{11}$, in which case $D \downarrow_{G}$ is irreducible, giving (i)(f).

Case $n=12$. Here $S$ is $L_{2}(11), M_{11}$, or $M_{12}$. Checking the irreducible characters of $G$, we conclude that $\operatorname{dim} D \leq 320$. As $D$ does not lift to characteristic 0 , one of the following holds: (a) $p=3$ and $\operatorname{dim} D=16,144,288$ or 320 ; (b) $p=11$ and $\operatorname{dim} D=128$. However, $G$ has no irreducible $p$-Brauer characters of those degrees.

Case $n=13$. Here $S=L_{3}(3)$, whence $\operatorname{dim} D \leq 52$. As $D$ does not lift to characteristic 0 , we have $p=13, H=\hat{S}_{13}$, and $\operatorname{dim} D=32$. But $G$ does not have irreducible 13-Brauer characters of such degree.

Case $n=14$. Here $S=L_{2}(13)$, whence $\operatorname{dim} D \leq 14$, which contradicts Corollary 3.1

Case $n=15$. Here $S$ is $A_{7}$ or $A_{8}$. By Lemma [2.2, $32 \mid \operatorname{dim} D$. By checking the Brauer characters of $G$ we see that $\operatorname{dim} D=32,64, S=A_{8}$, and $p \neq 3,7$. 
But $D$ does not lift to characteristic 0 . By Theorem A and [32, Table III], this implies $p=5, H=\hat{S}_{15}$, and $\operatorname{dim} D=64$. Now if $c \in G$ is an element of order 3 corresponding to a 3-cycle in $S$, then $\varphi(c)=4$. On the other hand, $D \downarrow_{\hat{A}_{15}}$ is just the reduction modulo 5 of a complex basic spin module of $\hat{A}_{15}$, and so $\varphi(c)=-2$ (see e.g. [22 p. 465]), a contradiction.

Case $n=16$. Here $\pi(G)=\mathbb{Z}_{2}^{4}: A$ for some $A \leq G L_{4}(2)$. By Lemma 2.2 $2^{7} \mid \operatorname{dim} D$, so $|A| \geq 2^{10}$ using Corollary 3.1. Moreover, $A$ acts irreducibly on $\mathbb{Z}_{2}^{4}$ by an argument similar to the one in [22 p.461]. It follows that $A=A_{7}$ or $A_{8}$, and $A$ acts transitively on the non-trivial elements of $\mathbb{Z}_{2}^{4}$. This in turn implies that $K:=\pi^{-1}\left(\mathbb{Z}_{2}^{4}\right) \cong \mathbb{Z}_{2}^{5}$. Writing $\operatorname{dim} D=2^{7} \cdot a$ for an integer $a$, we have $1 \leq a \leq 4$ because of Corollary 3.1 Decompose $\varphi \downarrow_{K}=s \sum_{i=1}^{t} \lambda_{i}$, where $16 \geq t \geq 2$ and $\left\{\lambda_{1}, \ldots, \lambda_{t}\right\}$ is an $A$-orbit on the set of 16 linear characters of $K$ that are faithful on $Z$. Set $A_{0}:=\operatorname{Stab}_{A}\left(\lambda_{1}\right)$. Then $\varphi \downarrow_{G}=\theta \uparrow^{G}$, where $\theta$ is an irreducible Brauer character of degree $s$ of $K: A_{0}$ and $\theta \downarrow_{K}=s \lambda_{1}$. Analyzing possible subgroups $A_{0}$ of $A$ and dimensions of modules over them, we see that $\theta \uparrow^{G}$ cannot have dimension of the form $2^{7} \cdot a$ as above.

Cases $n=17$ and $n=21$. Here $S=L_{2}(16)$ and $L_{3}(4)$, respectively, and we argue as in the case $n=14$.

Case $n=22$. Here $S=M_{22}$. By Lemma 2.2, $2^{9} \mid \operatorname{dim} D$. If $U$ is a composition factor of $D \downarrow_{G}$, then, since $\operatorname{Out}(S)=\mathbb{Z}_{2}$, we see that $2^{8} \mid \operatorname{dim} U$. But $G$ has no such irreducible representation $U$.

Similarly we can deal with the cases $n=23$ (where $S=M_{23}$ ), $n=24$ (where $S=M_{24}$ ), and $n=32$ (where $\mathbb{Z}_{2}^{5} \triangleleft H \leq A G L_{5}(2)$ ).

B. Now we assume that the $\mathbb{F} H$-module $D$ lifts to characteristic 0 . So $D$ is a reduction modulo $p$ of a complex irreducible spin representation $V$ of $H$. As $D \downarrow_{G}$ is irreducible so is $V \downarrow_{G}$, and we can apply Theorems 6.3 and 6.4 of [22], which describe all such pairs $(V, G)$. It remains to check whether reduction modulo $p$ of $V \downarrow_{G}$ is irreducible for each such pair. Denote this reduction by $V_{p}$.

B1. Suppose $H=\hat{S}_{n}$. Here $V$ is always a basic spin representation.

Case $n=8$. Then $\pi(G)=A G L_{3}(2)$. Using analysis of the case $n=8$ in $\mathbf{A}$, we conclude that $K \cong \mathbb{Z}_{2}^{4}$ and $G$ permutes transitively the 8 linear characters of $K$ that occur on $V$. This implies that $V_{p}$ is irreducible (as $\left.p \neq 2\right)$, giving the exception (i) (d).

Case $n=10$. By [22, p. 463], we have $\pi^{-1}(S)=\mathbb{Z}_{2} \times A_{6}$. If $p>5$, then $p \nmid|G|$, and we get the exceptions in (i)(e). On the other hand, if $p=3$ or 5 , then $G$ does not have irreducible modules of dimension 16 .

Case $n=12$. This leads to the exception (i)(g).

B2. Suppose $H=\hat{A}_{n}$.

Case $n=8$. Then $\pi(G)=A G L_{3}(2)$ and $V$ is either basic or second basic. The basic module lifts to $\hat{S}_{n}$, so (ii)(d) follows from the case $n=8$ in B1. Let $V$ be second basic. Then $p \neq 7$, as $V$ reduces modulo 7 . Adopt the notation of the case $n=8$ in $\mathbf{A}$. Then $K \cong \mathbb{Z}_{2}^{4}$ and $G$ acts transitively on the 8 linear characters of $K$ that occur on $V$. Note that $A_{0}$ acts on $V_{0}$, the $\lambda_{1}$-homogeneous component of dimension 3 , as $\mathbb{Z}_{2} \times\left(\mathbb{Z}_{7}: \mathbb{Z}_{3}\right)$, with $\mathbb{Z}_{3}$ cyclically permuting 3 non-trivial linear characters of $\mathbb{Z}_{7}$ on $V_{0}$. Hence $V_{p}$ is irreducible for $p \neq 7$, giving (iii)(b).

Case $n=9$. Here $V$ is basic and either $\mathbb{Z}_{2}^{3}: Q_{8} \leq \pi(G) \leq \mathbb{Z}_{2}^{3}: S L_{2}(3)$ or $S=L_{2}(8)$. In the former case $V_{3}$ is reducible, as otherwise $\mathbb{Z}_{3}^{2}$ would act trivially 
on $V_{3}$, contrary to the faithfulness. If $p>3$, then $p$ is coprime to $|G|$, leading to part of (ii)(e). The rest of (ii)(e) comes from checking the characters of $L_{2}(8)$ and its automorphism group.

Case $n=10$ leads to the exception (ii)(f) (except for the case $p \neq 5$, which has been covered in case $\mathbf{A}$ ).

Case $n=11$ leads to the exception (ii)(h).

Case $n=12$ leads to the exceptions (ii)(i) and (iii)(c).

The proof of Theorem B is complete.

\section{Nearly simple subgroups (Theorem C)}

We rely on the following result of Kleidman and Wales:

Proposition 6.1 ([22, 6.1]). Assume that $S$ is a non-abelian proper simple subgroup of $A_{n}$ and that $|\operatorname{Aut}(S)| \geq 2^{n-4}$. Then one of the following holds:

(a) $S \cong A_{m}$ for some $m \geq 9$ and each orbit of $S$ on $\Omega$ has length 1 or $m$.

(b) $S \cong A_{m}$ with $m=5,6,7$ or 8 , and $n \leq 10,14$, 16, or 19, respectively.

(c) $S \cong L_{2}(q)$ with $q=7,8,11,13$, or 16 , and $n \leq 12,14,14$, 15, or 17 , respectively.

(d) $S \cong L_{3}(3)$ or $L_{3}(4)$, and $n \leq 17$ or 21 , respectively.

(e) $S \cong M_{t}$ with $t=11,12,22,23$, or 24 , and $n \leq 16,21,23,27$, or 31 , respectively.

Let $H=\hat{S}_{n}$ or $\hat{A}_{n}$, and $D$ be a faithful $\mathbb{F} H$-module which remains irreducible on restriction to a nearly simple subgroup $G$. Then $\pi(G)$ is almost simple, because $Z(G)$ acts as scalar matrices on $D$. Moreover, by Corollary 3.1, we may assume that the socle $S$ of $\pi(G)$ is among the subgroups listed in Proposition 6.1. Finally, in view of Theorem B, we may assume that $\pi(G)$ is imprimitive on $\Omega$ (cf. Theorem $\mathrm{C}(\mathrm{iv})$ ). The following result of Phillips will help us to deal with intransitive subgroups. The proposition treats the irreducible restrictions of non-basic modules over $\hat{S}_{n}$ and $\hat{A}_{n}$ to Young type subgroups. The basic modules are treated in Corollary 4.2. The terminology used in Proposition 6.2 is explained in the introduction.

Proposition $6.2([29])$. Let $H=\hat{S}_{n}$ or $\hat{A}_{n}, D$ be a non-basic faithful irreducible $\mathbb{F} H$-module. Suppose $\mu=\left(\mu_{1}, \mu_{2}, \ldots\right)$ is a non-trivial composition of $n$, and $G$ is a subgroup in $H$ satisfying

$$
A_{\mu_{1}} \times A_{\mu_{2}} \times \cdots \leq \pi(G) \leq S_{\mu_{1}} \times S_{\mu_{2}} \times \cdots .
$$

Then the restriction $D \downarrow_{G}$ is irreducible if and only if one of the following happens:

(i) $H=\hat{S}_{n}, D=D(\lambda, \sigma)$, and one of the following happens:

(a) $G=\hat{S}_{n-1}, \lambda \in J S$, and $\lambda \in J S(0)$ if $a(\lambda)$ is even.

(b) $G=\hat{A}_{n-1}, \lambda \in J S(0)$, and $a(\lambda)$ is odd.

(c) $\pi(G)=S_{n-2} \times S_{2}, \lambda \in J S(0)$, and $\lambda_{[0]} \in J S(1)$.

(d) $\pi(G)=\left(S_{n-2} \times S_{2}\right) \cap A_{n} \cong S_{n-2}, \lambda \in J S(0), a(\lambda)$ is odd, and $\lambda_{[0]} \in J S(1)$.

(e) $G=\hat{S}_{n-2}, \lambda \in J S(0), a(\lambda)$ is odd, and $\lambda_{[0]} \in J S(1)$.

(ii) $H=\hat{A}_{n}, D=E(\lambda, \sigma)$, and one of the following happens:

(a) $G=\hat{A}_{n-1}, \lambda \in J S$, and $\lambda \in J S(0)$ if $a(\lambda)$ is odd.

(b) $\pi(G)=\left(S_{n-2} \times S_{2}\right) \cap A_{n} \cong S_{n-2}, \lambda \in J S(0)$, and $\lambda_{[0]} \in J S(1)$.

(c) $G=\hat{A}_{n-2}, \lambda \in J S(0), a(\lambda)$ is even, and $\lambda_{[0]} \in J S(1)$. 
Now, we can deal with a major part of Theorem C.

Proposition 6.3. Let $H=\hat{S}_{n}$ or $\hat{A}_{n}, D$ be a faithful irreducible $\mathbb{F} H$-module, and $G$ be a subgroup of $H$ such that $\pi(G)<S_{n}$ is an almost simple subgroup with $S:=\operatorname{soc}(\pi(G)) \cong A_{m}$ for some $5 \leq m<n$. Assume that each orbit of $S$ on $\Omega$ has length 1 or $m$. Then $D \downarrow_{G}$ is irreducible if and only if one of the cases (i)-(iii) of Theorem $C$ occurs.

Proof. If $H, G, D$ are as in (i)-(iii) of Theorem C, then the restriction is irreducible by Proposition 6.2 and Corollary 4.2, using the description of the partition $\omega_{n}$, which labels the basic spin module over $\mathbb{F} H$, given in (1.2). Conversely, let $D \downarrow_{G}$ be irreducible for $G$ as in the assumption.

We claim that there is only one $S$-orbit of length $m$ and other orbits are of length 1. Indeed, otherwise $\pi(G)$ is contained in a Young subgroup of the form $S_{m} \times S_{m} \times \ldots$. If $D$ is non-basic, it follows from Proposition 6.2 that the restriction is reducible. If $D$ is basic, then using Corollary 4.2 we conclude that $H=\hat{S}_{n}$ and one of the following happens:

(1) $p \nmid n=2 m, S<S_{m} \times S_{m}$;

(2) $p \mid n=2 m+1, S<S_{m} \times S_{m} \times S_{1}$.

However, in both cases $\pi(G)$ belongs to the subgroup $A_{\mu}:=S_{\mu} \cap A_{n}$, and the restriction to (the double cover of) such subgroup is reducible by Corollary 4.2 again.

So $\pi(G)$ is actually itself a Young type subgroup, i.e. $G$ satisfies the assumptions of Proposition 6.2. The result now follows by applying Proposition 6.2 and Corollary 4.2, and (1.2).

Now we complete the proof of Theorem $\mathrm{C}$ by going through the remaining cases listed in Proposition 6.1(b)-(e). If $(H, G, D)$ is as in Theorem C(iv)-(viii), then one readily checks using [12] and [19] that $D \downarrow_{G}$ is irreducible. Conversely, let $D \downarrow_{G}$ be irreducible, and $G$ be as in Proposition 6.1 (b)-(e) but not satisfy the assumptions of Proposition 6.3. Denote by $\mu(G)$ the largest dimension of an irreducible $\mathbb{F} G$ representation. Then, of course, we have

$$
\mu(G) \geq \delta(H) \geq \delta\left(\hat{A}_{n}\right) .
$$

We now claim that $S$ has exactly one non-trivial orbit on $\Omega$, on which it acts primitively. For groups listed in Proposition 6.1(c)-(e) this is clear, because the minimal index $P(S)$ of proper subgroups of $S$ is bigger than $n / 2$ (see [12]). Let $S$ be as in Proposition 6.1(b) and $S$ have more than one non-trivial orbit on $\Omega$. Then the upper bound on $n$ implies that $S \cong A_{m}(5 \leq m \leq 8)$ has exactly two orbits of length $m$ and $n-2 m$ fixed points on $\Omega$. So we can apply Proposition 6.3. Thus $S$ has exactly one non-trivial orbit $\Omega_{1}$, and we can use [12] again to conclude that $S$ must be primitive on this orbit. Let $s=\left|\Omega_{1}\right|$. Define

$$
K=\pi^{-1}\left(\bigcap_{j \in \Omega-\Omega_{1}} \operatorname{Stab}_{\pi(H)}(j)\right) .
$$

Now, we consider the groups appearing in (b)-(e) of Proposition 6.1 one by one. For the cases appearing in (b) we will assume that $s>m$, as otherwise the situation is covered by Proposition 6.3. In all cases we will assume that $s<n$, as otherwise $\pi(G)$ is primitive on $\Omega$. 
Case 1: $S=A_{5}$. Here $s=6$. As $\mu(G) \leq 6$, we have $n \leq 7$, thanks to (6.1). So $n=7$ and $G$ is of type $(6,1)$. Using the fact that $\pi(G)$ is primitive on $\Omega_{1}$ and Theorem B, we arrive at the cases listed in Theorem $\mathrm{C}(\mathrm{v})$.

Case 2: $S=A_{6}$. As $\mu\left(\hat{A}_{6} \cdot 2_{2}\right) \leq 10$, we have $\mu(G) \leq 20$, so $n \leq 12$ by (6.1). Suppose $n=12$. Since $\delta\left(\hat{A}_{12}\right)=32$ for $p \neq 3$, we have $p=3$. Moreover, $\operatorname{dim}(D) \leq$ 20 implies that $H=\hat{A}_{12}, D$ is basic spin, and $\operatorname{dim}(D)=16$. If $D \downarrow_{G}$ is irreducible and $U$ is any composition factor of $D \downarrow_{\hat{S}}$, then the dimension of $U$ must divide 16 . It follows that $\operatorname{dim}(U)=2$ or 4 , whence $U$ extends to $\hat{S} .2_{2}$. This in turn implies that $\operatorname{dim}(D) \leq 8$, a contradiction. Now, let $n \leq 11$. Then $s=10, n=11, \pi(G)$ is of type $(10,1)$ and $\pi(G)$ is contained in $\pi(K)$ as a primitive subgroup. Applying Theorem B and Corollary 4.2 leads to the cases in Theorem C(vi).

Case 3: $S=A_{7}$. Here $\mu(G) \leq 36$, so $n \leq 14$ by (6.1). Hence $s=7$, and so there are no cases to consider (as we have assumed that $s>m$ ).

Case 4: $S=A_{8}$. Here $\mu(G) \leq 112$, hence $n \leq 15$ by (6.1). As we have assumed $m<s<n$, there are no cases to consider.

Case 5: $S=L_{2}(7)$. Here $\mu(G) \leq 8$, hence $n \leq 10$ by (6.1) and $s=7$ or 8 . Moreover, $\delta\left(\hat{A}_{8}\right)=8$, so $\operatorname{dim}(D)=8$ and $D$ is basic spin. First suppose that $s=8$. In this case $\hat{S}<\hat{A}_{8}$, and $D \downarrow_{\hat{A}_{8}}$ lifts to a complex representation, say $W$. According to [22, p. 462], $W \downarrow_{\hat{S}}$ has two irreducible constituents, of degrees 1 and 7. This implies that $D \downarrow_{G}$ is reducible. Next suppose that $s=7$. Clearly, $\pi(G)$ acts on the $S$-orbit $\Omega_{1}$ of length 7 , but $L_{2}(7) .2$ has no subgroup of index 7 . Therefore $\pi(G)=S$ and $G$ is contained in $K$ as a primitive subgroup. By Theorem B applied to $(K, G, D), \operatorname{dim}(D)=4$ or 6 , a contradiction.

Case 6: $S=L_{2}(8)$. Here $\mu(G) \leq 27$, hence $n \leq 12$ by (6.1) and $s=9$. Suppose $n=11$ or 12 . Then $\operatorname{dim}(D) \geq \delta\left(\hat{A}_{11}\right)=16$, but $\pi(G)=S$ or $S .3$. Therefore $\operatorname{dim}(D)=21$ or 27 , which is a contradiction, as $H$ has no irreducible representations of such degree. Hence $n=10$, and $G$ is contained in $K=\hat{A}_{9}$ as a primitive subgroup. By Theorem B applied to $(K, G, D), D$ is basic spin of dimension 8. This leads to Theorem $\mathrm{C}($ vii).

Case 7: $S=L_{2}(11)$, or $L_{2}(13)$. Here $\mu(G) \leq 14$, hence $n \leq 10$ by (6.1). On the other hand, $n \geq P(S) \geq 11$, a contradiction.

Case 8: $S=L_{2}(16)$, or $L_{3}(4)$. In this case $n \leq P(S)$, whence $\pi(G)$ is primitive on $\Omega$, a contradiction.

Case 9: $S=L_{3}(3)$. Here $n \leq 17$ and so $s=13$. Since $L_{3}(3) .2$ cannot have an orbit of length $13, \pi(G)=S$. Thus $G$ is contained in $K=\hat{A}_{13}$ as a primitive subgroup. This is impossible by Theorem B applied to $(K, G, D)$.

Case 10: $S=M_{11}$. Here $n \leq 16$ and so $s=11$ or 12 . Moreover $\pi(G)=S$, therefore $G$ is contained in $K=\overline{\hat{A}}_{s}$ as a primitive subgroup. By Theorem B applied to $(K, G, D), s=11, p \neq 3$, and $\operatorname{dim}(D)=16$. But $n>s$, so $\operatorname{dim}(D) \geq \delta\left(\hat{A}_{12}\right)=$ 32 , a contradiction.

Case 11: $S=M_{12}$. Here $n \leq 21$ and so $s=12$. As $M_{12} .2$ cannot have an orbit of length $12, \pi(G)=S$. Thus $G$ is contained in $K=\hat{A}_{12}$ as a primitive subgroup. By Theorem B applied to $(K, G, D)$, either $p \neq 3$ and $\operatorname{dim}(D)=32$, or $p \neq 3,11$ or $\operatorname{dim}(D)=160$. The first case leads to Theorem $\mathrm{C}$ (viii). The second case is impossible, as $K<\hat{A}_{13} \leq H$ and $\hat{A}_{13}$ has no irreducible representation of degree 160 . 
Case 12: $S=M_{22}, M_{23}$, or $M_{24}$. If $S=M_{22}$, then $n \leq 23=P(S)+1$, whence $s=22$ and $\pi(G)$ is of type $(22,1)$. If $S=M_{23}$ or $M_{24}$, then $\pi(G)=S$ and $s=23$, resp. 24. In all cases $G$ is contained in $K$ as a primitive subgroup. This is impossible by Theorem B applied to $(K, G, D)$.

The proof of Theorem $\mathrm{C}$ is complete.

\section{Arbitrary subgroups (Theorem D)}

In this section we consider restrictions of non-basic modules over $\hat{S}_{n}$ and $\hat{A}_{n}$ to arbitrary subgroups. For information on basic modules see $\$ 4$ In most results of this section we have to assume that $p>3$. Recall the notation $\hat{g}$ from $\$ 2$.

Lemma 7.1. Let $p>3, n \geq 6$, and $C$ be the elementary abelian subgroup of order 8 in $S_{n}$ generated by the transpositions $(1,4),(2,5)$, and $(3,6)$. Set

$$
x:=\sum_{c \in C}(\operatorname{sgn} c) \hat{c}\left(1+t_{1} t_{2}+t_{2} t_{1}\right) \hat{c}^{-1} \in \mathbb{F} \hat{A}_{n} .
$$

Then $x D \neq 0$ for any non-basic faithful irreducible $\mathbb{F} \hat{A}_{n}$-module $D$.

Proof. If we prove the lemma for $n=6$, then the general case will follow by induction on $n$ using Lemma 2.4. So let $n=6$. First, we consider the case $\mathbb{F}=\mathbb{C}$. Let $V$ be an irreducible faithful $\mathbb{C} \hat{A}_{6}$-module not isomorphic to a basic spin module. It suffices to show that the $\operatorname{trace}^{\operatorname{tr}_{V}}\left(x t_{1} t_{2}\right)$ of the element $x t_{1} t_{2} \in \mathbb{C} \hat{A}_{6}$ on $V$ is a non-zero integer not divisible by $p$. Indeed, this implies that $x$ does not annihilate any reduction $\bar{V}$ of $V$ modulo $p$. If $p>5$, then $p$ does not divide the order of $\hat{A}_{6}$, so any irreducible module looks like $\bar{V}$. In fact, for faithful modules the same is true even for $p=5$; see 12 , 19 .

Finally, we prove that $\operatorname{tr}_{V}\left(x t_{1} t_{2}\right)$ is a non-zero integer not divisible by $p$. Note that $x=\sum_{c \in C}(\operatorname{sgn} c) \hat{c}\left(t_{1} t_{2}+t_{2} t_{1}\right) \hat{c}^{-1}$. We will write ${ }^{c} y$ for $\hat{c} y \hat{c}^{-1}, c \in S_{n}, y \in \mathbb{F} \hat{S}_{n}$. By conjugating with $t_{1} t_{2} t_{4} t_{5}$, we note that the elements ${ }^{c}\left(t_{1} t_{2}\right) t_{1} t_{2}$ are conjugate to each other for

$$
c \in\{(14),(2,5),(3,6)\} \quad \text { and } \quad c \in\{(1,4)(2,5),(1,4)(3,6),(2,5)(3,6)\} .
$$

The same is true for the elements of the form ${ }^{c}\left(t_{2} t_{1}\right) t_{1} t_{2}$. So, if $\chi$ is the character of $V$, then

$$
\begin{aligned}
\operatorname{tr}_{V}\left(x t_{1} t_{2}\right)= & \chi\left(t_{2} t_{1}\right)+\chi(1)-3 \chi\left(^{(1,4)}\left(t_{1} t_{2}\right) t_{1} t_{2}\right)-3 \chi\left({ }^{(1,4)}\left(t_{2} t_{1}\right) t_{1} t_{2}\right) \\
& +3 \chi\left({ }^{(1,4)(2,5)}\left(t_{1} t_{2}\right) t_{1} t_{2}\right)+3 \chi\left({ }^{(1,4)(2,5)}\left(t_{2} t_{1}\right) t_{1} t_{2}\right) \\
& -\chi\left(\left(^{(1,4)(2,5)(3,6)}\left(t_{1} t_{2}\right) t_{1} t_{2}\right)-\chi\left(^{(1,4)(2,5)(3,6)}\left(t_{2} t_{1}\right) t_{1} t_{2}\right) .\right.
\end{aligned}
$$

To identify the corresponding conjugacy classes it suffices to show, using relations, that

(i) $t_{2} t_{1}$ is the lift of $(1,3,2) \in S_{6}$ of order 3 ;

(ii) $t_{2} t_{1} t_{1} t_{2}=1$;

(iii) ${ }^{(1,4)}\left(t_{1} t_{2}\right) t_{1} t_{2}=t_{2} t_{3} t_{1} t_{2}$ is a lift of $(1,3)(2,4) \in S_{6}$ (all such lifts are conjugate);

(iv) ${ }^{(1,4)}\left(t_{2} t_{1}\right) t_{1} t_{2}=t_{3} t_{1} t_{2} t_{1}$ is a lift of $(1,4,3) \in S_{6}$ of order 6 ;

(v) ${ }^{(1,4)(2,5)}\left(t_{1} t_{2}\right) t_{1} t_{2}=t_{1} t_{3} t_{4} t_{2}$ is a lift of $(1,2,4,5,3) \in S_{6}$ of order 10 ;

(vi) ${ }^{(1,4)(2,5)}\left(t_{2} t_{1}\right) t_{1} t_{2}=t_{1} t_{4} t_{3} t_{2}$ is a lift of $(1,2,5,4,3) \in S_{6}$ of order 10 ; 
(vii) $(1,4)(2,5)(3,6)\left(t_{1} t_{2}\right) t_{1} t_{2}=t_{1} t_{2} t_{4} t_{5}$ is a lift of $(1,2,3)(4,5,6) \in S_{6}$ of order 3 ;

(viii) ${ }^{(1,4)(2,5)(3,6)}\left(t_{2} t_{1}\right) t_{1} t_{2}=t_{1} t_{2} t_{5} t_{4}$ is a lift of $(1,2,3)(4,6,5) \in S_{6}$ of order 3 .

Now, using the known character values of $\hat{S}_{6}$ [12], we find that $\operatorname{tr}_{V}\left(x t_{1} t_{2}\right)$ equals $18,3,3,12$, and 12 for irreducible non-basic spin representations $V$ of $\hat{S}_{n}$ of dimensions $4,8,8,10$, and 10 , respectively.

Let $H=\hat{S}_{n}$ or $\hat{A}_{n}$. Recall the $\mathbb{F} H$-modules $M^{\lambda}$ from $\$ 2$ For an $\mathbb{F} H$-module $D$, we set

$$
\mathcal{H}_{j}(D):=\operatorname{Hom}_{\mathbb{F} H}\left(M^{(j, n-j)}, \operatorname{End}_{\mathbb{F}}(D)\right) \quad \text { and } \quad d_{j}(D):=\operatorname{dim} \mathcal{H}_{j}(D) .
$$

The following is a crucial technical result.

Theorem 7.2. Let $p>3, n \geq 6, H=\hat{S}_{n}$ or $\hat{A}_{n}$, and $D$ be a non-basic irreducible faithful $\mathbb{F} H$-module. Then $d_{3}(D)>d_{2}(D)$.

Proof. Note that $M^{(j, n-j)}$ is the permutation module on the set of unordered $j$ tuples $\left\{i_{1}, i_{2}, \ldots, i_{j}\right\}$ of different numbers $i_{1}, i_{2}, \ldots, i_{j} \in\{1,2, \ldots, n\}$. Set

$$
f: M^{(3, n-3)} \rightarrow M^{(2, n-2)},\left\{i_{1}, i_{2}, i_{3}\right\} \mapsto\left\{i_{1}, i_{2}\right\}+\left\{i_{1}, i_{3}\right\}+\left\{i_{2}, i_{3}\right\} .
$$

By [7] 3.1], $f$ is surjective. Hence $f^{*}: \mathcal{H}_{2}(D) \rightarrow \mathcal{H}_{3}(D)$ defined by $f^{*}(\psi)=\psi \circ f$ is injective. So it suffices to prove that $\operatorname{Im} f^{*} \neq \mathcal{H}_{3}(D)$. To show this, we construct an element $\varphi \in H_{3} \backslash \operatorname{Im} f^{*}$. Set $\varphi(\{1,2,3\})$ to be the element of $\operatorname{End}_{F}(D)$ which maps $v \in D$ to $\left(1+t_{1} t_{2}+t_{2}+t_{1}\right) v$. Moreover, if $\sigma \in H$ is the element with $\sigma(1)=i_{1}, \sigma(2)=$ $i_{2}, \sigma(3)=i_{3}$, then we set $\varphi\left(\left\{i_{1}, i_{2}, i_{3}\right\}\right)$ to be the element of $\operatorname{End}_{F}(D)$ which maps $v \in D$ to $\sigma\left(1+t_{1} t_{2}+t_{2} t_{1}\right) \sigma^{-1} v$. As any $\sigma \in G:=H \cap \hat{S}_{(3, n-3)}$ commutes with $\left(1+t_{1} t_{2}+t_{2} t_{1}\right), \varphi$ is a well defined $\mathbb{F} H$-homomorphism from $M^{(3, n-3)}=\operatorname{ind}_{G}^{H} \mathbf{1}_{G}$ to $\operatorname{End}_{F}(D)$.

Now assume for a contradiction that $\varphi \in \operatorname{Im} f^{*}$. Then $\varphi=f^{*}(\psi)=\psi \circ f$ for some $\psi \in H_{2}$. Let $C$ be as in Lemma 7.1. Set $e=\sum_{c \in C}(\operatorname{sgn} c) \hat{c}\{1,2,3\} \in M^{(3, n-3)}$. In view of [17, 4.10], we have $f(e)=0$, so $\varphi(e)=\psi(f(e))=0$. By definition of $\varphi$, this means that $x D=0$ for $x$ as in Lemma [7.1. But this contradicts Lemma [.1.

For $1 \leq k \leq n$ and a subgroup $G \leq S_{n}$, we set

$$
r_{k}(G)=\sharp\{G \text {-orbits on unordered } k \text {-element subsets of }\{1,2, \ldots, n\}\} \text {. }
$$

If $r_{k}(G)=1$, the group $G$ is called $k$-homogeneous. If $k \leq n / 2$, it is well known [28] that $r_{k}(G) \geq r_{k-1}(G)$ for any $G$. The following result shows that often $r_{3}(G)>$ $r_{2}(G)$.

Lemma 7.3 ([11, $\S 5$, Corollary]). Let $n>5, G<S_{n}$ be transitive, and $r_{2}(G)=$ $r_{3}(G)$. Then one of the following holds:

(i) $n$ is even and $G$ has 2 blocks of imprimitivity of size $n / 2$.

(ii) $n$ is even and $G$ has $n / 2$ blocks of imprimitivity of size 2 .

(iii) $G$ is 3-homogeneous.

Theorem 7.4. Let $p>3, n \geq 6, H=\hat{S}_{n}$ or $\hat{A}_{n}$, and $G<H$ be a subgroup. Assume that the restriction $D \downarrow_{G}$ is irreducible for some non-basic faithful $\mathbb{F} H$ module D. Then one of the following holds:

(i) $n$ is even, $\pi(G)$ is transitive and has 2 blocks of imprimitivity of size $n / 2$.

(ii) $n$ is even, $\pi(G)$ is transitive and has $n / 2$ blocks of imprimitivity of size 2 .

(iii) $\pi(G)$ is 3-homogeneous. 
(iv) $\pi(G)$ has an orbit of length $n-1$ or $n-2$ on $\{1,2, \ldots, n\}$, and $\pi(G)$ is 3-homogeneous on this orbit.

Proof. Suppose $\pi(G)$ does not satisfy the conditions (i)-(iv) above. There are three cases:

(1) $\pi(G)$ is transitive. Then $r_{3}(\pi(G))>r_{2}(\pi(G))$ by Lemma 7.3 .

(2) $\pi(G)$ is contained in a Young subgroup $S_{\lambda}$ or $A_{\lambda}$, call it $H_{\lambda}$, with $\lambda \neq$ $(n-1,1),(n-2,2),(n-2,1,1)$. One easily checks that we have $r_{3}\left(H_{\lambda}\right)>$ $r_{2}\left(H_{\lambda}\right)$.

(3) $\pi(G)$ is contained in a Young subgroup $H_{\lambda}$, with $\lambda \in\{(n-1,1)$, $(n-2,2),(n-2,1,1)\}$, but $\pi(G)$ is not 3 -homogeneous on the 'long' orbit. Here again we have $r_{3}(\pi(G))>r_{2}(\pi(G))$.

Thus, if we can show that the restriction to a subgroup $G$ with $r_{3}(\pi(G))>r_{2}(\pi(G))$ is reducible, the theorem will be proved (in case (2) we will have that already the restriction to $H_{\lambda}$ is reducible).

As $r_{k}(\pi(G))$ equals the dimension of the $G$-invariant space $\left(M^{(n-k, k)}\right)^{G}$ and $d_{3}(D)>d_{2}(D)$ by Theorem [7.2, the proof of Proposition 3.4 in [7] shows that $\operatorname{dim} \operatorname{End}_{\mathbb{F} G}\left(D \downarrow_{G}\right)>1$, and so the result follows from Schur's Lemma.

Remark. (i) In case (iii) of Theorem [7.4, $\pi(G)$ is primitive, and so this case is covered by Theorem B.

(ii) Proposition 6.2 proved in [29] shows that irreducible restrictions of a nonbasic spin module from $H$ to $H_{\lambda}$ are possible only if

$$
\lambda \in\{(n-1,1),(n-2,2),(n-2,1,1)\} .
$$

Theorem 7.4 provides an easier proof of this result, providing $p>3$.

To complete the proof of Theorem D, it remains to refine case (iv) of Theorem 7.4 We need the following proposition:

Proposition 7.5. Let $n \geq 5$, and $X<S_{n}$ be a 3-homogeneous subgroup with $\operatorname{soc}(X)$ not simple. Then one of the following holds:

(i) $X=A G L_{m}(2)$ with $n=2^{m}$;

(ii) $X=2^{4} . A_{7}$ with $n=16$;

(iii) $X=A G L_{1}(8)$ or $A \Gamma L_{1}(8)$ with $n=8$;

(iv) $X=A \Gamma L_{1}(32)$ with $n=32$.

Proof. The 3-homogeneous groups which are not 3-transitive were described in [20]. This yields the cases (iii) and (iv) above. Now we may assume that $X$ is 3 -transitive. Set $\Omega:=\{1,2, \ldots, n\}$. Since $\operatorname{soc}(X)$ is not simple, it follows from the O'Nan-Scott Theorem that $\operatorname{soc}(X)=Z_{q}^{m}$, for some prime $q$, and it acts regularly on $\Omega$. Then $X=V X_{0}$, where $V=Z_{q}^{m}$ and $X$ acts on $\Omega=V$ as affine transformations.

Now, $X_{0}$ acts 2-transitively on $V \backslash\{0\}$. On the other hand, if $q>2$ and $m>1$, or if $q>3$ and $m=1$, then $X_{0} \leq G L(V)$ acts imprimitively on $V \backslash\{0\}$. In our case $q^{m} \geq 5$. Hence $q=2$ and $X_{0} \leq G L_{m}(2)$. Now consider $Y:=\operatorname{soc}\left(X_{0}\right)$. We have two cases.

1) $Y$ is non-abelian simple. Since $N:=|V \backslash\{0\}|=2^{m}-1 \geq 7$, in view of the classification of 2-transitive groups (see [10, 21]), one of the following holds:

a) $Y=A_{N}$. This is impossible, as $A_{N}$ with $N=2^{m}-1$ cannot be embedded in $G L_{m}(2)$.

b) $Y=A_{7}$ and $N=15$. This leads to case (ii). 
c) $Y={ }^{2} B_{2}(q)$ and $N=q^{2}+1$. Impossible, as $N=2^{m}-1$.

d) $Y={ }^{2} A_{2}(q)$ and $N=q^{3}+1$. Impossible, as $N=2^{m}-1$.

e) $Y=P S L_{d}(q)$ and $N=\left(q^{d}-1\right) /(q-1)$. Assume $q$ is even. Then, since $N=2^{m}-1 \geq 7$, we see that $q=2, d=m, X_{0}=G L_{m}(2)$, and $G=A S L_{m}(2)$, giving case (i) of the proposition. Finally, let $q$ be odd. Since $N=2^{m}-1 \geq 7$, we see that $d \geq 3$ and $d$ is odd. Now, $Y=P S L_{d}(q)$ embeds into $G L_{m}(2)$, giving a cross-characteristic representation of $P S L_{d}(q)$. By 14, Corollary 6.2], we have $m \geq\left(q^{d}-1\right) /(q-1)-2$. This contradicts the equality $2^{m}-1=\left(q^{d}-1\right) /(q-1)$.

2) $Y$ is elementary abelian, i.e. $Y=Z_{r}^{k}$ for a prime $r$. Then $7 \leq 2^{m}-1=r^{k}$. An elementary number theory argument shows that $k=1$ and $r=2^{m}-1$. In this case $X_{0} \leq N_{G L_{m}(2)}\left(Z_{r}\right)=Z_{r} . Z_{m}$. In particular, $\left|X_{0}\right| \leq r m$. On the other hand, $X_{0}$ is 2-transitive on $V \backslash\{0\}$, so $\left|X_{0}\right| \geq r(r-1)$, a contradiction.

Now, let $G$ be as in (iv), and let $\Omega_{1}$ be the 'long' orbit. Assume that $D \downarrow_{G}$ is irreducible. Set $s:=\left|\Omega_{1}\right|$, and let $K$ be defined as in (6.2). We have $s=n-1$ or $n-2, K \cong \hat{S}_{s}$ if $H=\hat{S}_{n}$, and $K \cong \hat{A}_{s}$ if $H=\hat{A}_{n}$. Let $X<S_{s}$ be the image of $\pi(G)$ with respect to its action on $\Omega_{1}$. We apply Proposition 7.5 to the action of $X$ on $\Omega_{1}$. We have that $X$ is as in cases (i)-(iv) in Proposition 7.5. In particular, $X$ has no subgroups of index 2 .

We claim that $\pi(G)=X$ and $\pi(G) \leq \pi(K)$. This is obvious if $s=n-1$, or if $s=n-2$ and $\pi(G)$ is of type $(n-2,1,1)$. Assume $s=n-2$ and $\pi(G)$ is of type $(n-2,2)$. Then $\pi(G) \cap \pi(K)$ has index 2 in $\pi(G)$, and it embeds in $X$. But $X$ has no subgroups of index 2 , so $\pi(G) \cap \pi(K)=X$. We may assume $\pi(G)=\langle X, x\rangle$, where $x$ interchanges the two points of $\Omega \backslash \Omega_{1}$. Since $\pi(G) \cap \pi(K)=X$, there is $y \in \pi(G)$ that fixes $\Omega \backslash \Omega_{1}$ pointwise and induces the same action as $x$ on $\Omega_{1}$. Replacing $x$ by $x y^{-1}$, we may assume that $x$ is a transposition. Since $X$ has no subgroups of index $2, \hat{X}$ is contained in $\hat{A}_{s}$ and so it is centralized by $\hat{x}$. Thus $G$ is centralized by $\hat{x}$, a non-central involution in $\hat{S}_{n}$, so $D \downarrow_{G}$ is reducible, a contradiction.

Now we know that $G \leq K$, and the action of $\pi(G)$ on $\Omega_{1}$ is certainly primitive, not containing $A_{s}$. Moreover, as $D$ is non-basic, we have by Lemma 2.4 that the restriction $D \downarrow_{K}$ has a non-basic composition factor $D^{\prime}$. So we may apply Theorem B to $\left(K, G, D^{\prime}\right)$, and we also know that $\pi(G 0$ is as in (i)-(iv) of Proposition 7.5 We then get $s=8, m=3, \pi(G)=A G L_{3}(2), \operatorname{dim}(D)=24$. Here $n \geq 9$, so we get a contradiction, as $H \in \hat{A}_{9,10}, \hat{S}_{9,10}$ has no non-basic representation of degree 24 . The proof of Theorem D is complete.

\section{Tensor products (Theorem F)}

Recall the notation $d_{j}(V)$ introduced in (7.1).

Theorem 8.1. Let $p>3, H=\hat{S}_{n}$ or $\hat{A}_{n}$ with $n \geq 7$. Suppose $A, B$ are irreducible $\mathbb{F} H$-modules such that $d_{3}(A)>d_{2}(A)$ and $d_{3}(B)>d_{2}(B)$. Then the $H$-module $A \otimes B$ is reducible.

Proof. Consider the irreducible $\mathbb{F} S_{n}$-module $D_{j}$ labelled by $(n-j, j)$ with $j=0,1,2$, or 3. In particular, $D_{0}=\mathbf{1}$. It is well known that $D_{j}$ is self-dual, and the modules $D_{j} \downarrow_{A_{n}}$ are also irreducible and distinct. Throughout this proof we write $G=\pi(H)$ for short. The condition $d_{3}(A)>d_{2}(A)$ means that

$$
\operatorname{dim} \operatorname{Hom}_{\mathbb{F} H}\left(M^{(n-3,3)}, A \otimes A^{*}\right)>\operatorname{dim} \operatorname{Hom}_{\mathbb{F} H}\left(M^{(n-2,2)}, A \otimes A^{*}\right) .
$$


The proof of [7, 3.4] applied to $\operatorname{End}_{\mathbb{F}}(A) \cong A \otimes A^{*}$ shows that one of the following holds.

(I) There is no $r$ such that $1 \leq r \leq 3$ and $n \equiv 5-r(\bmod p)$. Then there is an injective $G$-homomorphism

$$
1 \oplus D_{3} \stackrel{\alpha}{\hookrightarrow} A \otimes A^{*} .
$$

(II) There is (a unique) $r$ such that $1 \leq r \leq 3$ and $n \equiv 5-r(\bmod p)$. Let $s:=3-r$. Then, in the notation of 7], one of the following holds:

(A1) There is an injective $G$-homomorphism

$$
\mathbf{1} \oplus\left(S^{(n-3,3)}\right)^{*}=\mathbf{1} \oplus D_{3} \mid D_{s} \stackrel{\alpha}{\hookrightarrow} A \otimes A^{*} .
$$

(A2) $r \neq 3$ and there is an injective $G$-homomorphism

$$
\mathbf{1} \oplus S^{(n-3,3)}=\mathbf{1} \oplus D_{s} \mid D_{3} \stackrel{\alpha}{\hookrightarrow} A \otimes A^{*} .
$$

(A3) $r=3$ and there is an injective $G$-homomorphism

$$
Y^{(n-3,3)}=\mathbf{1}\left|D_{3}\right| \mathbf{1} \stackrel{\alpha}{\hookrightarrow} A \otimes A^{*} .
$$

We label the corresponding cases for $B$ by (B1), (B2), (B3), respectively, and the corresponding $G$-homomorphism by $\beta$.

We aim to show that $\operatorname{dim} \operatorname{Hom}_{\mathbb{F} G}\left(B \otimes B^{*}, A \otimes A^{*}\right)>1$. As

$$
\operatorname{Hom}_{\mathbb{F} H}\left(B \otimes B^{*}, A \otimes A^{*}\right) \cong \operatorname{Hom}_{\mathbb{F} H}(A \otimes B, A \otimes B),
$$

this implies that $A \otimes B$ is not irreducible by Schur's Lemma. There is an obvious non-zero homomorphism $B \otimes B^{*} \rightarrow \mathbf{1} \hookrightarrow A \otimes A^{*}$. Here and below $\rightarrow$ means a surjective homomorphism. We show that there is another homomorphism $\gamma$ which is linearly independent from this one.

Suppose (I) holds. Then take $\gamma$ to be the map

$$
B \otimes B^{*} \stackrel{\beta^{*}}{\rightarrow}\left(\mathbf{1} \oplus D_{3}\right)^{*} \cong \mathbf{1} \oplus D_{3} \stackrel{\alpha}{\hookrightarrow} A \otimes A^{*} .
$$

Now suppose we are in the case (II).

If (A1) holds for $A$ and (B1) holds for $B$, take $\gamma$ to be the map

$$
B \otimes B^{*} \stackrel{\beta^{*}}{\rightarrow}\left(\mathbf{1} \oplus D_{3} \mid D_{s}\right)^{*} \cong \mathbf{1} \oplus D_{s} \mid D_{3} \rightarrow D_{3} \stackrel{\alpha}{\hookrightarrow} A \otimes A^{*} .
$$

Now assume that $r=3$. Then $D_{s} \cong 1$. If (A3) holds for $A$ and (B3) holds for $B$, then take $\gamma$ to be the map

$$
B \otimes B^{*} \stackrel{\beta^{*}}{\rightarrow}\left(\mathbf{1}\left|D_{3}\right| \mathbf{1}\right)^{*} \cong \mathbf{1}\left|D_{3}\right| \mathbf{1} \stackrel{\alpha}{\hookrightarrow} A \otimes A^{*} .
$$

If (A1) holds for $A$ and (B3) holds for $B$, then take $\gamma$ to be the map

$$
B \otimes B^{*} \stackrel{\beta^{*}}{\rightarrow}\left(\mathbf{1}\left|D_{3}\right| \mathbf{1}\right)^{*} \cong \mathbf{1}\left|D_{3}\right| \mathbf{1} \rightarrow D_{3} \mid \mathbf{1} \stackrel{\alpha}{\hookrightarrow} A \otimes A^{*} .
$$

In the case of (A3) and (B1) we can interchange $A$ and $B$.

Finally, assume that $r \neq 3$. In this case $D_{s} \neq 1$. If (A2) holds for $A$ and (B2) holds for $B$, then take $\gamma$ to be the map

$$
B \otimes B^{*} \stackrel{\beta^{*}}{\rightarrow}\left(\mathbf{1} \oplus D_{s} \mid D_{3}\right)^{*} \cong \mathbf{1} \oplus D_{3} \mid D_{s} \rightarrow D_{s} \stackrel{\alpha}{\hookrightarrow} A \otimes A^{*} .
$$

If (A1) holds for $A$ and (B2) holds for $B$, then take $\gamma$ to be the map

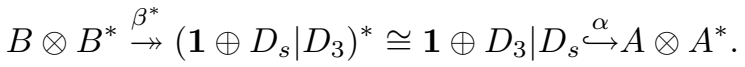

In the case of (A2) and (B1) we can interchange $A$ and $B$. 
Now Theorem F(i) follows from Theorems 7.2 and 8.1 , and Theorem F(ii) follows from Theorems [7.2, 8.1] and [7, 3.9].

\section{ACKNOWLEDGEMENT}

The authors are grateful to Jan Saxl for useful discussions.

\section{REFERENCES}

[1] M. Aschbacher, On the maximal subgroups of the finite classical groups, Invent. Math. 76 (1984), 469-514. MR 86a:20054

[2] A. Balog, C. Bessenrodt, J.B. Olsson and K. Ono, Prime power degree representations of the symmetric and alternating groups, J. London Math. Soc. (2) 64 (2001), 344-356. MR 2002g:20025

[3] C. Bessenrodt, On mixed products of complex characters of the double covers of the symmetric groups, Pacific J. Math. 199 (2001), 257-268. MR 2002d:20014

[4] C. Bessenrodt and A. Kleshchev, On Kronecker products of complex representations of the symmetric and alternating groups, Pacific J. Math. 190 (1999), 201-223. MR 2000i:20017

[5] C. Bessenrodt and A. Kleshchev, On tensor products of modular representations of symmetric groups, Bull. London Math. Soc. 32 (2000), 292-296. MR 2001a:20023

[6] C. Bessenrodt and A. Kleshchev, Irreducible tensor products over alternating groups, $J$. Algebra 228 (2000), 536-550. MR 2001b:20017

[7] J. Brundan and A. S. Kleshchev, Representations of the symmetric group which are irreducible over subgroups, J. reine angew. Math. 530 (2001), 145-190. MR 2001m:20017

[8] J. Brundan and A. S. Kleshchev, Projective representations of symmetric groups via Sergeev duality, Math. Z. 239 (2002), 27-68. MR 2003b:20018

[9] J. Brundan and A. Kleshchev, Hecke-Clifford superalgebras, crystals of type $A_{2 \ell}^{(2)}$ and modular branching rules for $\widehat{S}_{n}$, Represent. Theory 5 (2001), 317-403. MR 2002j:17024

[10] P.J. Cameron, Finite permutation groups and finite simple groups, Bull. London Math. Soc. 13 (1981), 1-22. MR 83m:20008

[11] P.J. Cameron, P.M. Neumann, and J. Saxl, An interchange property in finite permutation groups, Bull. London Math. Soc. 11(1979), 161-169. MR 80g:20005

[12] J.H. Conway, R.T. Curtis, S.P. Norton, R.A. Parker, R.A. Wilson, Atlas of Finite Groups, Clarendon Press, Oxford, 1985. MR 88g:20025

[13] R. Gow and A. Kleshchev, Connections between the representations of the symmetric group and the symplectic group in characteristic 2, J. Algebra 221 (1999), 60-89. MR 2001b:20020

[14] R.M. Guralnick and Pham Huu Tiep, Low-dimensional representations of special linear groups in cross characteristics, Proc. London Math. Soc. (3) 78 (1999), 116-138. MR 2000a:20016

[15] P. N. Hoffman and J. F. Humphreys, Projective Representations of the Symmetric Group, Clarendon Press, Oxford, 1992. MR 94f:20027

[16] I. M. Isaacs, Character Theory of Finite Groups, Academic Press, New York, 1976. MR 57:417

[17] G. D. James, The Representation Theory of the Symmetric Groups, Springer Lecture Notes 682, Berlin, Heidelberg, New York, 1978. MR 80g:20019

[18] J.C. Jantzen and G.M. Seitz, On the representation theory of the symmetric groups, Proc. London Math. Soc. (3) 65 (1992), 475-504. MR 93k:20026

[19] C. Jansen, K. Lux, R. A. Parker and R. A. Wilson, An Atlas of Brauer Characters, Oxford University Press, Oxford, 1995. MR 96k:20016

[20] W.M. Kantor, k-homogeneous groups, Math. Z. 124 (1972), 261-265. MR 46:5422

[21] W.M. Kantor, Homogeneous designs and geometric lattices, J. Combin. Theory (A) 38 (1985), 66-74. MR 87c:51007

[22] P. B. Kleidman and D. B. Wales, The projective characters of the symmetric groups that remain irreducible on subgroups, J. Algebra 138 (1991), 440-478. MR 92e:20008

[23] P. Kleidman and M. Liebeck, The Subgroup Structure of the Finite Classical Groups, London Mathematical Society Lecture Note Series, 129, Cambridge University Press, Cambridge, 1990. MR 91g:20001 
[24] A.S. Kleshchev, On restrictions of irreducible modular representations of semisimple algebraic groups and symmetric groups to some natural subgroups. I, Proc. London Math. Soc. (3) 69 (1994), 515-540. MR 95i:20065a

[25] A.S. Kleshchev and J. Sheth, Representations of the symmetric group are reducible over simply transitive subgroups, Math. Z. 235 (2000), 99-109. MR 2001i:20027

[26] A.S. Kleshchev and J. Sheth, Representations of the alternating group which are irreducible over subgroups, Proc. London Math. Soc. (3) 84 (2002), 194-212. MR 2002i:20012

[27] M.W. Liebeck and G.M. Seitz, On the subgroup structure of classical groups, Invent. Math. 134 (1998), 427-453. MR 99h:20074

[28] D. Livingstone and A. Wagner, Transitivity of finite permutation groups on unordered sets, Math. Z. 90 (1965), 393-403. MR 32:4183

[29] A. Phillips, Branching problems for projective representations of the symmetric and alternating groups, preprint, 2003.

[30] J. Saxl, The complex characters of the symmetric groups that remain irreducible in subgroups, J. Algebra 111 (1987), 210-219. MR 88i:20011

[31] A. Wagner, An observation on the degrees of projective representations of the symmetric and alternating groups over an arbitrary field, Arch. Math. 29 (1977), 583- 589. MR 57:444

[32] D. B. Wales, Some projective representations of $S_{n}$, J. Algebra 61 (1979), 37-57. MR 81f:20015

Department of Mathematics, University of Oregon, Eugene, Oregon 97403

E-mail address: klesh@math.uoregon.edu

Department of Mathematics, University of Florida, Gainesville, Florida 32611

E-mail address: tiep@math.ufl.edu 